\title{
FACES OF HIGHEST WEIGHT MODULES AND THE UNIVERSAL WEYL POLYHEDRON
}

\author{
GURBIR DHILLON AND APOORVA KHARE
}

\begin{abstract}
Let $V$ be a highest weight module over a Kac-Moody algebra $\mathfrak{g}$, and let conv $V$ denote the convex hull of its weights. We determine the combinatorial isomorphism type of conv $V$, i.e. we completely classify the faces and their inclusions. In the special case where $\mathfrak{g}$ is semisimple, this brings closure to a question studied by Cellini and Marietti $[\operatorname{IMRN} 2015]$ for the adjoint representation, and by Khare [J. Algebra 2016; Trans. Amer. Math. Soc. 2017] for most modules. The determination of faces of finite-dimensional modules up to the Weyl group action and some of their inclusions also appears in previous works of Satake [Ann. of Math. 1960], Borel and Tits [IHES Publ. Math. 1965], Vinberg [Izv. Akad. Nauk 1990], and Casselman [Austral. Math. Soc. 1997].

For any subset of the simple roots, we introduce a remarkable convex cone which we call the universal Weyl polyhedron, which controls the convex hulls of all modules parabolically induced from the corresponding Levi factor. Namely, the combinatorial isomorphism type of the cone stores the classification of faces for all such highest weight modules, as well as how faces degenerate as the highest weight gets increasingly singular. To our knowledge, this cone is new in finite and infinite type.

We further answer a question of Michel Brion, by showing that the localization of conv $V$ along a face is always the convex hull of the weights of a parabolically induced module. Finally, as we determine the inclusion relations between faces representation-theoretically from the set of weights, without recourse to convexity, we answer a similar question for highest weight modules over symmetrizable quantum groups.
\end{abstract}

\section{Contents}

1. Introduction

2. Statement of results 4

Acknowledgments

Date: August 18, 2017.

2010 Mathematics Subject Classification. Primary: 17B10; Secondary: 17B67, 22E47, 17B37, 20G42, 52B20.

Key words and phrases. Highest weight module, parabolic Verma module, Weyl polytope, integrable Weyl group, $f$-polynomial, universal Weyl polyhedron. 
3. Notation and preliminaries

4. Classification of faces of highest weight modules 13

5. Inclusions between faces 17

6. Families of Weyl polyhedra and representability 21

7. Faces of the universal Weyl polyhedron 23

8. Weak families of Weyl polyhedra and concavity 30

9. Closure, polyhedrality, and integrable isotropy

10. A question of Brion on localization of convex hulls 39

11. Highest weight modules over symmetrizable quantum groups 40 References

\section{INTRODUCTION}

Throughout the paper, unless otherwise specified $\mathfrak{g}$ is a Kac-Moody algebra over $\mathbb{C}$ with triangular decomposition $\mathfrak{n}^{-} \oplus \mathfrak{h} \oplus \mathfrak{n}^{+}$and Weyl group $W$, and $V$ is a $\mathfrak{g}$-module of highest weight $\lambda \in \mathfrak{h}^{*}$.

In this paper we study and answer several fundamental convexity-theoretic questions related to the weights of $V$ and their convex hull, denoted wt $V$ and conv $V$ respectively. In contrast to previous approaches in the literature, our approach is to use techniques from representation theory to directly study wt $V$ and deduce consequences for conv $V$.

To describe our results and the necessary background, first suppose $\mathfrak{g}$ is of finite type and $V$ is integrable. In this case, conv $V$ is a convex polytope, called the Weyl polytope, and is well understood. In particular, the faces of such Weyl polytopes have been determined by Vinberg [26, §3] and Casselman [6, §3], and as Casselman notes, are implicit in the earlier work of Satake [25, §2.3] and Borel-Tits [3, \$12.16]. The faces are constructed as follows. Let $I$ index the simple roots of $\mathfrak{g}$, and $e_{i}, f_{i}, h_{i}, i \in I$ the Chevalley generators of $\mathfrak{g}$. Given $J \subset I$, denote by $\mathfrak{l}_{J}$ the corresponding Levi subalgebra generated by $e_{j}, f_{j}, \forall j \in J$ and $h_{i}, \forall i \in I$. Also define the following distinguished set of weights:

$$
\operatorname{wt}_{J} V:=\operatorname{wt} U\left(\mathfrak{l}_{J}\right) V_{\lambda},
$$

where $V_{\lambda}$ is the highest weight space. The aforementioned authors showed that every face of the Weyl polytope for $V$ is a Weyl group translate of the convex hull of wt $J V$ for some $J \subset I$.

To complete the classification of faces, it remains to determine the redundancies in the above description. Formally, consider the face map:

$$
\mathrm{F}_{V}: W \times 2^{I} \rightarrow 2^{\mathrm{wt} V}, \quad \mathrm{~F}_{V}(w, J):=w\left(\mathrm{wt}_{J} V\right),
$$


where $2^{S}$ denotes the power set of a set $S$. As wt $J V$ and its convex hull determine one another, computing the redundancies is the same as computing the fibers of the face map. The determination of the fibers for the restriction $\mathrm{F}_{V}(1,-)$ is implicit in the above works of Vinberg and Casselman. A complete understanding of the fibers of $\mathrm{F}_{V}(-,-)$ was achieved very recently by Cellini-Marietti [7] for the adjoint representation, and subsequently, by Khare [18] for all highest weight modules. While the fibers of the face map were understood for all highest weight modules $V$, it was not known whether all faces were of the above form.

In this paper we resolve both parts of the above problem for any highest weight module $V$ over a Kac-Moody algebra $\mathfrak{g}$. While the answer takes a similar form to the known cases in finite type, the proof of the first part requires a substantially different argument, as prior methods fail at several crucial junctures:

(i) A key input in the known cases of the classification of faces of conv $V$ was that every face is the maximizer of a linear functional, which was deduced from polyhedrality. However, even the polyhedrality of conv $V$ was not known in general in finite type.

(ii) We show in related work [8] that indeed conv $V$ is always a polyhedron in finite type. However as we show below, for $\mathfrak{g}$ of infinite type, conv $V$ is rarely polyhedral, generically locally polyhedral, and in some cases not locally polyhedral. In particular, one cannot a priori appeal to a maximizing functional to understand a face.

(iii) Finally, even given a face cut out by a maximizing functional, the possibility of the functional to fall outside the Tits cone makes the usual analysis inapplicable.

In this paper we provide an infinitesimal analysis of the faces that avoids invocation of functionals, completing the first part of the classification of faces. As a consequence, every face is a posteriori cut out by a maximizing functional lying in the Tits cone. For the second part, i.e. computing the redundancies, we give a direct representation theoretic argument which in particular simplifies the existing arguments in finite type.

Moreover, we construct a remarkable convex cone, which we call the universal Weyl polyhedron $\mathcal{P}_{\text {univ }}$, whose combinatorial isomorphism class controls the convex hulls of weights of all highest weight modules which are parabolically induced from a fixed Levi. Namely, (i) the classification of faces of $\mathcal{P}_{\text {univ }}$ is equivalent to that of all such highest weight modules, and (ii) inclusions of faces of $\mathcal{P}_{\text {univ }}$ control how faces of such highest weight modules degenerate as the highest weight specializes. 


\section{Statement of Results}

Let $\mathfrak{g}$ be a Kac-Moody algebra and $V$ a highest weight $\mathfrak{g}$-module. We now state the first part of the classification of faces of conv $V$.

Theorem 2.1. Denote by $I_{V}$ the integrability of $V$, i.e.:

$$
I_{V}:=\left\{i \in I: f_{i} \text { acts locally nilpotently on } V\right\} \text {. }
$$

For each standard Levi subalgebra $\mathfrak{l}_{J} \subset \mathfrak{g}$, the locus conv $U\left(\mathfrak{l}_{J}\right) V_{\lambda}$ is a face of conv $V$. These account for every face of $\operatorname{conv} V$ which contains an interior point in the $I_{V}$ dominant chamber. An arbitrary face $F$ of conv $V$ is in the $W_{I_{V}}$ orbit of a unique such face.

For $\mathfrak{g}$ of finite type, this classification was obtained by Satake 25], Borel-Tits [3], Vinberg [26], and Casselman [6] for $V$ finite-dimensional, and by Khare [17] for many infinite-dimensional modules. Theorem 2.1 settles the remaining cases in finite type, and is to our knowledge new in infinite type. As explained above, a proof in this setting requires new ideas. Interestingly, our proof, which proceeds by induction on the rank of $\mathfrak{g}$, uses the consideration of non-integrable modules in an essential way, even for the case of integrable modules.

The classification of inclusion relations between faces, akin to the one in finite type [7, 18], is our next main result. By Theorem 2.1, it suffices to study the fibers of the face map $\mathrm{F}_{V}: W_{I_{V}} \times 2^{I} \rightarrow 2^{\mathrm{wt} V}$, defined as in (1.2).

Theorem 2.2. For each $J \subset I$ there exist sets $J_{\min } \subset J \subset J_{\max } \subset I$ such that:

$$
\begin{array}{ll}
\text { wt }_{J} V \subset \text { wt }_{J^{\prime}} V & \Longleftrightarrow J_{\min } \subset J^{\prime}, \\
\text { wt }_{J} V=\text { wt }_{J^{\prime}} V & \Longleftrightarrow J_{\min } \subset J^{\prime} \subset J_{\max } .
\end{array}
$$

In particular, in the poset $2^{I}$ under inclusion, the fibers of the face map $\mathrm{F}_{V}(1,-)$ are intervals. Moreover, the fibers of $\mathrm{F}_{V}(-, J)$ are left cosets of $W_{I_{V} \cap J_{\max }}$ in $W_{I_{V}}$. Given the final assertion of Theorem [2.1, this determines the fibers of the map $\mathrm{F}_{V}(-,-)$.

Explicit formulas for $J_{\min }, J_{\max }$ are presented before Theorem 5.1 and after Remark 5.5. respectively. In particular, we determine the $f$-polynomial of conv $V$, cf. Proposition 5.14. Thus, Theorems 2.1 and 2.2 together settle the classification problem for faces of arbitrary highest weight modules, over all Kac-Moody algebras.

Modulo the action of the Weyl group, Theorems 2.1 and 2.2 are essentially statements about the actions of the standard Levi subalgebras on the highest weight line. In this light, they admit a natural extension to quantum groups, which we prove in Section 11.

As shown below, the sets $J_{\min }, J_{\max }$, and therefore the combinatorial isomorphism class of conv $V$, only depend on $\lambda$ through $\left\{i \in I_{V}:\left(\check{\alpha}_{i}, \lambda\right)=0\right\}$. Motivated by this, we study deformations of the convex hulls conv $V$ for highest weight modules 
with fixed integrability. To interpolate between such convex hulls, we fix $J \subset I$, and consider Weyl polyhedra, which are simply convex combinations of the convex hulls of highest weight modules with integrability $J$. We study a natural notion of a family of Weyl polyhedra over a convex base $S$, and show that such families admit a classifying space.

Theorem 2.3. Consider the contravariant functor $\mathrm{F}$ from convex sets to sets, which takes a convex set to all families of Weyl polyhedra over it, and morphisms to pullbacks of families. Then $\mathrm{F}$ is representable.

If $B_{\text {univ }}$ represents $\mathrm{F}$, we obtain a universal family of Weyl polyhedra $\mathcal{P}_{\text {univ }} \rightarrow$ $B_{\text {univ }}$. Concretely, one may take $B_{\text {univ }}$ to be a parabolic analogue of the dominant chamber, so that $\mathcal{P}_{\text {univ }} \rightarrow B_{\text {univ }}$ extends the usual parametrization of modules by their highest weight. We study the convex geometry of the total space $\mathcal{P}_{\text {univ }}$, and show a tight connection between its combinatorial isomorphism type and the previous results on the classification of faces.

Theorem 2.4. For $J \subset I$ as above, $\mathcal{P}_{\text {univ }}$ is a convex cone. Write $\mathcal{F}$ for the set of faces of $\mathcal{P}_{\text {univ }}$. Then $\mathcal{F}$ can be partitioned:

$$
\mathcal{F}=\bigsqcup_{J^{\prime} \subset J} \mathcal{F}_{J^{\prime}}
$$

so that each $\mathcal{F}_{J^{\prime}}$, ordered by inclusion, is isomorphic to the face poset of conv $V$, where $V$ is any highest weight module with integrability $J$ and highest weight $\lambda$ with $J^{\prime}=\left\{j \in J:\left(\check{\alpha}_{j}, \lambda\right)=0\right\}$.

We in fact show more than is stated in Theorem 2.4 the remaining inclusions of faces are determined by how faces of $\operatorname{conv} V$ degenerate as the highest weight specializes, cf. Proposition 7.14. In particular, we determine the $f$-polynomial of $\mathcal{P}_{\text {univ }}$, cf. Proposition 7.15. We are unaware of a precursor to Theorem 2.4 or its aforementioned strengthening in the literature.

Theorem 2.4 is proved by using the projection to $B_{\text {univ }}$. More precisely, along the interior of each face of $B_{\text {univ }}$, the restriction of $\mathcal{P}_{\text {univ }}$ is a 'fibration', and we use this to construct faces of $\mathcal{P}_{\text {univ }}$ from 'constant' families of faces along the fibres.

Finally, we explain a question of Michel Brion on localization of faces, which we answer in this paper.

To do so, we introduce a notion of localization in convex geometry. If $E$ is a real vector space, and $C \subset E$ a convex subset, then for $c \in C$ to study the 'germ' of $C$ near $c$ one may form the tangent cone $T_{c} C$, given by all rays $c+t v, v \in E, t \in \mathbb{R}^{\geqslant 0}$ such that for $0<t \ll 1, c+t v \in C$. Given a face $F$ of $C$, one can similarly form the 'germ' of $C$ along $F$, by setting $\operatorname{Loc}_{F} C$ to be the intersection of the tangent cones $T_{f} C, \forall f \in F$. It may be helpful to think of tangent cones as convex analogues of tangent spaces and $\operatorname{Loc}_{F} C$ a convex analogue of the normal bundle of a submanifold. 
Let $\mathfrak{g}$ be of finite type, $\lambda$ a regular dominant integral weight, and $L(\lambda)$ the corresponding simple highest weight module. Brion observed that for any face $F$ of conv $L(\lambda), \operatorname{Loc}_{F}$ conv $L(\lambda)$ is always the convex hull of a parabolic Verma module for a Category $\mathcal{O}$ corresponding to the triangular decomposition $w \mathfrak{n}^{-} w^{-1} \oplus \mathfrak{h} \oplus w \mathfrak{n}^{+} w^{-1}$, for some $w \in W$. Further, if $\lambda \in F$, then one could take $w=e$. Brion asked whether this phenomenon persists for a general highest weight module $V$.

In answering Brion's question we will prove the equivalence of several convexitytheoretic properties of the convex hull of a highest weight module, which we feel is of independent interest. In particular, the following result explains the subtlety in classifying faces of conv $V$ for $\mathfrak{g}$ of infinite type, as alluded to in the introduction.

Theorem 2.5. Let $\mathfrak{g}$ be indecomposable of infinite type and $V$ a highest weight $\mathfrak{g}$-module. Then conv $V$ is a polytope if and only if $V$ is one-dimensional, and is otherwise a polyhedron if and only if $I_{V}$ corresponds to a Dynkin diagram of finite type. If $V$ is not one-dimensional, the following are equivalent:

(1) The intersection of conv $V$ with any polytope is a polytope.

(2) The tangent cone $T_{\lambda} V$ is closed, and conv $V=\bigcap_{w \in W_{I_{V}}} T_{w \lambda} V$.

(3) conv $V$ is closed.

(4) $\lambda$ lies in the interior of the $I_{V}$ Tits cone.

In the somewhat unrepresentative case of integrable modules in finite type, Theorem 2.5 is straightforward and well known, see e.g. [14, §2.3]. For general $\mathfrak{g}$ and $V$, one of the implications can be deduced from work of Looijenga on root systems 22], and a theorem concerning local polyhedrality in a similar geometric context appears in his later work [23]. Otherwise, to our knowledge Theorem 2.5 is largely new, including for all non-integrable modules in finite type.

Returning to Brion's question, given Theorem 2.1 it suffices to consider faces of the form conv $U\left(\mathfrak{l}_{J}\right) V_{\lambda}$. We show:

Theorem 2.6. Suppose $\lambda$ has trivial stabilizer in $W_{I_{V}}$, and $J \subset I$. Writing $F$ for the face conv $U\left(\mathfrak{l}_{J}\right) V_{\lambda}$, we have:

$$
\operatorname{Loc}_{F} \operatorname{conv} V=\operatorname{conv} M\left(\lambda, I_{V} \cap J\right) .
$$

The regularity assumption appearing in Theorem 2.6 specializes to that of Brion for integrable modules in finite type. That such a condition is necessary to recover from conv $V$ the convex hull of a module with less integrability is already clear from considering edges, i.e. the case of $\mathfrak{s l}_{2}$.

In Brion's motivating example, this convexity-theoretic localization is a combinatorial shadow of localizing the corresponding line bundle on the flag variety off of 
certain Schubert divisors. As we develop in [8], for general $V$ with dot-regular highest weight in finite type, the above formula is the combinatorial shadow of localizing the corresponding (twisted) $D$-module on the flag variety.

Organization of the paper. After discussing preliminaries and establishing notation in Section 3, we show in Sections 4, 5, 6, 7, 9, 10 in order the main results stated above, Theorems 2.12 2.6. In Section 8 we prove an analogue of Theorem 2.3 for a more flexible notion of Weyl polyhedra, and in Section 11 we show the analogue of Theorem 2.2 for $U_{q}(\mathfrak{g})$-modules.

\section{ACKNOWLEDGMENTS}

We thank Michel Brion, Daniel Bump, and Victor Reiner for valuable discussions. The work of G.D. is partially supported by the Department of Defense (DoD) through the NDSEG fellowship. A.K. is partially supported by an Infosys Young Investigator Award.

\section{Notation AND PRELIMINARIES}

We now set notation and recall preliminaries and results from the companion work [8]; in particular, the material in this section and Section 11.1 overlaps with loc. cit. We advise the reader to skim Subsections 3.2 and 3.5, and refer back to the rest only as needed.

Write $\mathbb{Z}$ for the integers, and $\mathbb{Q}, \mathbb{R}, \mathbb{C}$ for the rational, real, and complex numbers respectively. For a subset $S$ of a real vector space $E$, write $\mathbb{Z} \geqslant 0 S$ for the set of finite linear combinations of $S$ with coefficients in $\mathbb{Z}^{\geqslant 0}$, and similarly $\mathbb{Z} S, \mathbb{Q} \geqslant 0 S, \mathbb{R} S$, etc.

3.1. Notation for Kac-Moody algebras, standard parabolic and Levi subalgebras. The basic references are [13] and [19]. In this paper we work throughout over $\mathbb{C}$. Let $I$ be a finite set. Let $A=\left(a_{i j}\right)_{i, j \in I}$ be a generalized Cartan matrix, i.e., an integral matrix satisfying $a_{i i}=2, a_{i j} \leqslant 0$, and $a_{i j}=0$ if and only if $a_{j i}=0$, for all $i \neq j \in I$. Fix a realization, i.e., a triple $(\mathfrak{h}, \pi, \check{\pi})$, where $\mathfrak{h}$ is a complex vector space of dimension $|I|+\operatorname{corank}(A)$, and $\pi=\left\{\alpha_{i}\right\}_{i \in I} \subset \mathfrak{h}^{*}, \check{\pi}=\left\{\check{\alpha}_{i}\right\}_{i \in I} \subset \mathfrak{h}$ are linearly independent collections of vectors satisfying $\left(\check{\alpha}_{i}, \alpha_{j}\right)=a_{i j}, \forall i, j \in I$. We call $\pi$ and $\check{\pi}$ the simple roots and simple coroots, respectively.

Let $\mathfrak{g}:=\mathfrak{g}(A)$ be the associated Kac-Moody algebra generated by $\left\{e_{i}, f_{i}: i \in I\right\}$ and $\mathfrak{h}$, modulo the relations:

$$
\begin{aligned}
{\left[e_{i}, f_{j}\right]=} & \delta_{i j} \check{\alpha}_{i}, \quad\left[h, e_{i}\right]=(h, \alpha) e_{i}, \quad\left[h, f_{i}\right]=-(h, \alpha) f_{i}, \quad[\mathfrak{h}, \mathfrak{h}]=0, \quad \forall h \in \mathfrak{h}, i, j \in I, \\
& \left(\operatorname{ad} e_{i}\right)^{1-a_{i j}}\left(e_{j}\right)=0, \quad\left(\operatorname{ad} f_{i}\right)^{1-a_{i j}}\left(f_{j}\right)=0, \quad \forall i, j \in I, i \neq j .
\end{aligned}
$$


Denote by $\overline{\mathfrak{g}}(A)$ the quotient of $\mathfrak{g}(A)$ by the largest ideal intersecting $\mathfrak{h}$ trivially; these coincide when $A$ is symmetrizable. When $A$ is clear from context, we will abbreviate these to $\mathfrak{g}, \overline{\mathfrak{g}}$.

In the following we establish notation for $\mathfrak{g}$; the same apply for $\overline{\mathfrak{g}}$ mutatis mutandis. Let $\Delta^{+}, \Delta^{-}$denote the sets of positive and negative roots, respectively. We write $\alpha>0$ for $\alpha \in \Delta^{+}$, and similarly $\alpha<0$ for $\alpha \in \Delta^{-}$. For a sum of roots $\beta=\sum_{i \in I} k_{i} \alpha_{i}$ with all $k_{i} \geqslant 0$, write $\operatorname{supp} \beta:=\left\{i \in I: k_{i} \neq 0\right\}$. Write

$$
\mathfrak{n}^{-}:=\bigoplus_{\alpha<0} \mathfrak{g}_{\alpha}, \quad \mathfrak{n}^{+}:=\bigoplus_{\alpha>0} \mathfrak{g}_{\alpha} .
$$

Let $\leqslant$ denote the standard partial order on $\mathfrak{h}^{*}$, i.e. for $\mu, \lambda \in \mathfrak{h}^{*}, \mu \leqslant \lambda$ if and only if $\lambda-\mu \in \mathbb{Z}^{\geqslant 0} \pi$.

For any $J \subset I$, let $\mathfrak{l}_{J}$ denote the associated Levi subalgebra generated by $\left\{e_{i}, f_{i}\right.$ : $i \in J\}$ and $\mathfrak{h}$; denote $\mathfrak{l}_{i}:=\mathfrak{l}_{\{i\}}$ for $i \in I$. For $\lambda \in \mathfrak{h}^{*}$ write $L_{\mathfrak{l}_{J}}(\lambda)$ for the simple $\mathfrak{l}_{J}$-module of highest weight $\lambda$. Writing $A_{J}$ for the principal submatrix $\left(a_{i, j}\right)_{i, j \in J}$, we may (non-canonically) realize $\mathfrak{g}\left(A_{J}\right)=: \mathfrak{g}_{J}$ as a subalgebra of $\mathfrak{g}(A)$. Now write $\pi_{J}, \Delta_{J}^{+}, \Delta_{J}^{-}$for the simple, positive, and negative roots of $\mathfrak{g}\left(A_{J}\right)$ in $\mathfrak{h}^{*}$, respectively (note these are independent of the choice of realization). Finally, we define the associated Lie subalgebras $\mathfrak{u}_{J}^{+}, \mathfrak{u}_{J}^{-}, \mathfrak{n}_{J}^{+}, \mathfrak{n}_{J}^{-}$by:

$$
\mathfrak{u}_{J}^{ \pm}:=\bigoplus_{\alpha \in \Delta^{ \pm} \backslash \Delta_{J}^{ \pm}} \mathfrak{g}_{\alpha}, \quad \mathfrak{n}_{J}^{ \pm}:=\bigoplus_{\alpha \in \Delta_{J}^{ \pm}} \mathfrak{g}_{\alpha},
$$

and $\mathfrak{p}_{J}:=\mathfrak{l}_{J} \oplus \mathfrak{u}_{J}^{+}$to be the associated parabolic subalgebra.

3.2. Weyl group, parabolic subgroups, Tits cone. Write $W$ for the Weyl group of $\mathfrak{g}$, generated by the simple reflections $\left\{s_{i}, i \in I\right\}$, and let $\ell: W \rightarrow \mathbb{Z}^{\geqslant 0}$ be the associated length function. For $J \subset I$, let $W_{J}$ denote the parabolic subgroup of $W$ generated by $\left\{s_{j}, j \in J\right\}$.

Write $P^{+}$for the dominant integral weights, i.e. $\left\{\mu \in \mathfrak{h}^{*}:\left(\check{\alpha}_{i}, \mu\right) \in \mathbb{Z}^{\geqslant 0}, \forall i \in I\right\}$. The following choice is non-standard. Define the real subspace $\mathfrak{h}_{\mathbb{R}}^{*}:=\left\{\mu \in \mathfrak{h}^{*}\right.$ : $\left.\left(\check{\alpha}_{i}, \mu\right) \in \mathbb{R}, \forall i \in I\right\}$. Now define the dominant chamber as $D:=\left\{\mu \in \mathfrak{h}^{*}:\left(\check{\alpha}_{i}, \mu\right) \in\right.$ $\left.\mathbb{R}^{\geqslant 0}, \forall i \in I\right\} \subset \mathfrak{h}_{\mathbb{R}}^{*}$, and the Tits cone as $C:=\bigcup_{w \in W} w D$.

Remark 3.1. In [19] and [13], the authors define $\mathfrak{h}_{\mathbb{R}}^{*}$ to be a real form of $\mathfrak{h}^{*}$. This is smaller than our definition whenever the generalized Cartan matrix $A$ is noninvertible, and has the consequence that the dominant integral weights are not all in the dominant chamber, unlike for us. This is a superficial difference, but our convention helps avoid constantly introducing arguments like [19, Lemma 8.3.2].

We will also need parabolic analogues of the above. For $J \subset I$, define $\mathfrak{h}_{\mathbb{R}}^{*}(J):=$ $\left\{\mu \in \mathfrak{h}^{*}:\left(\check{\alpha}_{j}, \mu\right) \in \mathbb{R}, \forall j \in J\right\}$, the $J$ dominant chamber as $D_{J}:=\left\{\mu \in \mathfrak{h}^{*}:\left(\check{\alpha}_{j}, \mu\right) \in\right.$ 
FACES OF HIGHEST WEIGHT MODULES AND THE UNIVERSAL WEYL POLYHEDRON 9

$\left.\mathbb{R}^{\geqslant 0}, \forall j \in J\right\}$, and the $J$ Tits cone as $C_{J}:=\bigcup_{w \in W_{J}} w D_{J}$. Finally, we write $P_{J}^{+}$for the $J$ dominant integral weights, i.e. $\left\{\mu \in \mathfrak{h}^{*}:\left(\check{\alpha}_{j}, \mu\right) \in \mathbb{Z}^{\geqslant 0}, \forall j \in J\right\}$. The following standard properties will be used without further reference in the paper; the reader can easily check that the standard arguments, cf. [19, Proposition 1.4.2], apply in this setting mutatis mutandis.

Proposition 3.2. For $\mathfrak{g}(A)$ with realization $(\mathfrak{h}, \pi, \check{\pi})$, let $\mathfrak{g}\left(A^{t}\right)$ be the dual algebra with realization $\left(\mathfrak{h}^{*}, \check{\pi}, \pi\right)$. Write $\check{\Delta}_{J}^{+}$for the positive roots of the standard Levi subalgebra $\mathfrak{l}_{J}^{t} \subset \mathfrak{g}\left(A^{t}\right)$.

(1) For $\mu \in D_{J}$, the isotropy group $\left\{w \in W_{J}: w \mu=\mu\right\}$ is generated by the simple reflections it contains.

(2) The $J$ dominant chamber is a fundamental domain for the action of $W_{J}$ on the $J$ Tits cone, i.e., every $W_{J}$ orbit in $C_{J}$ meets $D_{J}$ in precisely one point.

(3) $C_{J}=\left\{\mu \in \mathfrak{h}_{\mathbb{R}}^{*}(J):(\check{\alpha}, \mu)<0\right.$ for at most finitely many $\left.\check{\alpha} \in \check{\Delta}_{J}^{+}\right\}$; in particular, $C_{J}$ is a convex cone.

(4) Consider $C_{J}$ as a subset of $\mathfrak{h}_{\mathbb{R}}^{*}(J)$ in the analytic topology, and fix $\mu \in C_{J}$. Then $\mu$ is an interior point of $C_{J}$ if and only if the isotropy group $\left\{w \in W_{J}\right.$ : $w \mu=\mu\}$ is finite.

We also fix $\rho \in \mathfrak{h}^{*}$ satisfying $\left(\check{\alpha}_{i}, \rho\right)=1, \forall i \in I$, and define the dot action of $W$ via $w \cdot \mu:=w(\mu+\rho)-\rho$. Recall this does not depend on the choice of $\rho$. Indeed, for any $w \in W$, a standard induction on $\ell(w)$ shows:

$$
w(\rho)-\rho=\sum_{\alpha>0: w \alpha<0} w(\alpha) .
$$

We define the dot dominant chamber to be $\dot{D}:=\left\{\mu \in \mathfrak{h}^{*}:\left(\check{\alpha}_{i}, \mu+\rho\right) \in \mathbb{R}^{\geqslant 0}, \forall i \in\right.$ $I\}$, and the dot Tits cone to be $\bigcup_{w \in W} w \cdot \dot{D}$. It is clear how to similarly define their parabolic analogues: the $J$ dot dominant chamber and the $J$ dot Tits cone. Now Proposition 3.2 holds for the dot action, mutatis mutandis.

3.3. Representations, integrability, and parabolic Verma modules. Given an $\mathfrak{h}$-module $M$ and $\mu \in \mathfrak{h}^{*}$, write $M_{\mu}$ for the corresponding simple eigenspace of $M$, i.e. $M_{\mu}:=\{m \in M: h m=(h, \mu) m \forall h \in \mathfrak{h}\}$, and write wt $M:=\left\{\mu \in \mathfrak{h}^{*}: M_{\mu} \neq 0\right\}$.

Now recall $V$ is a highest weight $\mathfrak{g}$-module with highest weight $\lambda \in \mathfrak{h}^{*}$. For $J \subset I$, write wt $V:=$ wt $U\left(\mathfrak{l}_{J}\right) V_{\lambda}$, i.e. the weights of the $\mathfrak{l}_{J}$-module generated by the highest weight line. We say $V$ is $J$ integrable if $f_{j}$ acts locally nilpotently on $V$, $\forall j \in J$. Let $I_{V}$ denote the maximal $J$ for which $V$ is $J$ integrable, i.e.,

$$
I_{V}=\left\{i \in I:\left(\check{\alpha}_{i}, \lambda\right) \in \mathbb{Z}^{\geqslant 0}, f_{i}^{\left(\check{\alpha}_{i}, \lambda\right)+1} V_{\lambda}=0\right\} .
$$

We will call $W_{I_{V}}$ the integrable Weyl group. 
We next remind the basic properties of parabolic Verma modules over Kac-Moody algebras. These are also known in the literature as generalized Verma modules, e.g. in the original papers by Lepowsky (see 20] and the references therein).

Fix $\lambda \in \mathfrak{h}^{*}$ and a subset $J$ of $I_{L(\lambda)}=\left\{i \in I:\left(\check{\alpha}_{i}, \lambda\right) \in \mathbb{Z}^{\geqslant 0}\right\}$. The parabolic Verma module $M(\lambda, J)$ co-represents the following functor from $\mathfrak{g}$-mod to Set:

$$
M \rightsquigarrow\left\{m \in M_{\lambda}: \mathfrak{n}^{+} m=0, f_{j} \text { acts nilpotently on } m, \forall j \in J\right\} .
$$

When $J$ is empty, we simply write $M(\lambda)$ for the Verma module. Explicitly:

$$
M(\lambda, J) \simeq M(\lambda) /\left(f_{j}^{\left(\check{\alpha}_{j}, \lambda\right)+1} M(\lambda)_{\lambda}, \forall j \in J\right) .
$$

Finally, it will be useful to us to recall the following result on the weights of integrable highest weight modules, i.e. those $V$ with $I_{V}=I$.

Proposition 3.7 ([13, $\S 11.2$ and Proposition 11.3(a)]). For $\lambda \in P^{+}, \mu \in \mathfrak{h}^{*}$, say $\mu$ is non-degenerate with respect to $\lambda$ if $\mu \leqslant \lambda$ and $\lambda$ is not perpendicular to any connected component of $\operatorname{supp}(\lambda-\mu)$. Let $V$ be an integrable module of highest weight $\lambda$.

(1) If $\mu \in P^{+}$, then $\mu \in \mathrm{wt} V$ if and only if $\mu$ is non-degenerate with respect to $\lambda$.

(2) If the sub-diagram on $\left\{i \in I:\left(\check{\alpha}_{i}, \lambda\right)=0\right\}$ is a disjoint union of diagrams of finite type, then $\mu \in P^{+}$is non-degenerate with respect to $\lambda$ if and only if $\mu \leqslant \lambda$.

(3) wt $V=\left(\lambda-\mathbb{Z}^{\geqslant 0} \pi\right) \cap \operatorname{conv}(W \lambda)$.

3.4. Convexity. Let $E$ be a finite-dimensional real vector space (we will take $E=$ $\left.\mathfrak{h}^{*}\right)$. For a subset $X \subset E$, write conv $X$ for its convex hull, and aff $X$ for its affine hull. For an $\mathfrak{h}$-module $M$, for brevity we write conv $M$, aff $M$, for conv wt $M$, aff wt $M$, respectively. A collection of vectors $v_{0}, \ldots, v_{n}$ is said to be affine independent if their affine hull has dimension $n$. A closed half space is a locus of the form $\zeta^{-1}([t, \infty))$, for $\zeta \in E^{*}, t \in \mathbb{R}$. For us, a polyhedron is a finite intersection of closed half spaces, and a polytope is a compact polyhedron. The following well known alternative characterization of polyhedra will be useful to us:

Proposition 3.8 (Weyl-Minkowski). A subset $X \subset E$ is a polyhedron if and only if it can be written as the Minkowski sum $X=\operatorname{conv}\left(X^{\prime}\right)+\mathbb{R}^{\geqslant 0} X^{\prime \prime}$, for two finite subsets $X^{\prime}, X^{\prime \prime}$ of $E$. If $X$ is a polyhedron that does not contain an affine line, then $X^{\prime}, X^{\prime \prime}$ as above of minimal cardinality are unique up to rescaling vectors in $X^{\prime \prime}$ by $\mathbb{R}^{>0}$.

Let $C \subset E$ be convex. We endow aff $C$ with the analytic topology, and denote by relint $C$ the interior of $C$, viewed as a subset of aff $C$; this is always non-empty. For brevity we will often refer to $c \in \operatorname{relint} C$ as an interior point of $C$. For a point 
$c \in C$, we denote the tangent cone at $c$ by $T_{c} C$; recall this is the union of the rays $c+\mathbb{R}^{\geqslant 0}\left(c^{\prime}-c\right), \forall c^{\prime} \in C$. Given a highest weight module $V$ and $\mu \in$ wt $V$, we will write $T_{\mu} V$ for $T_{\mu}$ (conv wt $V$ ) for convenience.

A convex subset $F \subset C$ is called a face if whenever a convex combination $\sum_{i} t_{i} c_{i}$ of points $c_{i}, 1 \leqslant i \leqslant n$ of $C$ lies in $F$, all the points $c_{i}$ lie in $F$. Two convex sets $C, C^{\prime} \subset E$ are said to be combinatorially isomorphic if there is a dimensionpreserving isomorphism of their face posets, ordered by inclusion.

If $\zeta$ is a real linear functional, and $\left.\zeta\right|_{C}$ has a maximum value $m$, the locus $\{c \in$ $C:(\zeta, c)=m\}$ is an exposed face. All exposed faces are faces, but the reverse implication does not hold in general. The following simple lemmas will be used later.

Lemma 3.9. If $F$ is a face of $C$, then $F=C \cap$ aff $F$.

Lemma 3.10. If $F_{1}, F_{2}$ are faces of $C$, and $F_{1}$ contains a point of relint $F_{2}$, then $F_{1}$ contains $F_{2}$. In particular, if relint $F_{1} \cap$ relint $F_{2} \neq \emptyset$, then $F_{1}=F_{2}$.

Lemma 3.11. If $F$ is a face of $C$ and $\lambda \in F$, then $T_{\lambda} F$ is a face of $T_{\lambda} C$.

By a rational structure on $E$, we mean the choice of a rational subspace $P_{\mathbb{Q}} \subset E$ such that $P_{\mathbb{Q}} \otimes_{\mathbb{Q}} \mathbb{R} \stackrel{\sim}{\rightarrow} E$. Let us fix a rational structure $P$ on $E=\mathfrak{h}^{*}$, such that $\mathbb{Q} \pi \subset P_{\mathbb{Q}}$. With the choice of rational structure, one can make sense of rational vectors, rational hyperplanes and half-spaces, and by taking finite intersections of rational half-spaces, rational polyhedra. We have the following:

Proposition 3.12 (Basic properties of rational polyhedra).

(1) The affine hull of a rational polyhedron is rational.

(2) A cone is rational if and only if it is generated by rational vectors.

(3) A polytope is rational if and only if its vertices are rational points.

(4) A polyhedron is rational if and only if it is the Minkowski sum of a rational polytope and a rational cone.

(5) Every face of a rational polyhedron is a rational polyhedron.

(6) Every point in a rational polyhedron is a convex combination of rational points.

Proof. (1)-(5) may be found in [5, Proposition 1.69]. For the last point, by (5) and the supporting hyperplane theorem it suffices to consider an interior point; by (1) it suffices to note that if $U$ is an open set in $\mathbb{R}^{n}$ containing a point $q$, we can find $q_{i} \in U \cap \mathbb{Q}^{n}$ such that $q$ is a convex combination of the $q_{i}$; one may in fact choose them so that aff $q_{i}=\mathbb{R}^{n}$.

3.5. Results from previous work on highest weight modules. We now recall results from recent work [8] on the structure of wt $V$ for an arbitrary highest weight 
$\mathfrak{g}$-module $V$. The first result explains how every module $V$ is equal up to "first order" to its parabolic Verma cover $M\left(\lambda, I_{V}\right)$. In particular, we obtain the convex hull of wt $V$ :

Theorem 3.13. The following data are equivalent:

(1) $I_{V}$, the integrability of $V$.

(2) $\operatorname{conv} V$, the convex hull of the weights of $V$.

(3) The stabilizer of conv $V$ in $W$.

In particular, the convex hull in (2) is always that of the parabolic Verma module $M\left(\lambda, I_{V}\right)$, and the stabilizer in (3) is always the parabolic subgroup $W_{I_{V}}$.

The argument for the equality of conv $M\left(\lambda, I_{V}\right)$ and conv $V$ involves two ingredients that are repeatedly used in this paper. The first is to study the weights of $M\left(\lambda, I_{V}\right)$ via restriction to the Levi subalgebra $\mathfrak{l}_{I_{V}}$ corresponding to $I_{V}$. The representation decomposes into a direct sum corresponding to cosets of $\mathfrak{h}^{*}$ modulo translation by $\Delta_{I_{V}}$, the roots of $\mathfrak{l}_{I_{V}}$, and it transpires each 'slice' has easily understood weights:

Proposition 3.14 (Integrable Slice Decomposition, [8]). For $J \subset I_{L(\lambda)}$, we have:

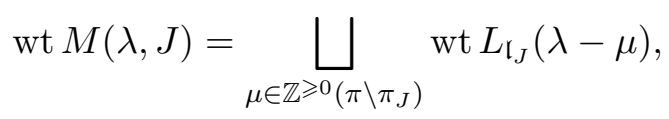

where $L_{\mathfrak{l}_{J}}(\nu)$ denotes the simple $\mathfrak{l}_{J}$-module of highest weight $\nu$. In particular, wt $M(\lambda, J)$ lies in the J Tits cone (cf. Section 3.2). Moreover,

$$
\text { wt } M(\lambda, J)=(\lambda+\mathbb{Z} \pi) \cap \operatorname{conv} M(\lambda, J) .
$$

We include two illustrations of Equation (3.15) in Figure 3.1.

Next, using Proposition 3.14 we obtain a formula for conv $M\left(\lambda, I_{V}\right)$ via 'generators', i.e. the convex hull of an explicit set of points and rays, which also lie in conv $V$.

Proposition 3.17 (Ray Decomposition, [8]).

$$
\operatorname{conv} V=\operatorname{conv} \bigcup_{w \in W_{I_{V}}, i \in I \backslash I_{V}} w\left(\lambda-\mathbb{Z}^{\geqslant 0} \alpha_{i}\right) .
$$

When $I_{V}=I$, by the right-hand side we mean conv $\bigcup_{w \in W} w \lambda$. Moreover, each ray $w\left(\lambda-\mathbb{R}^{\geqslant 0} \alpha_{i}\right)$ is a face of conv $V$.

We include two illustrations of Proposition 3.17 in Figure 3.2. To our knowledge Proposition 3.17 was not known in either finite or infinite type prior to [8]. 

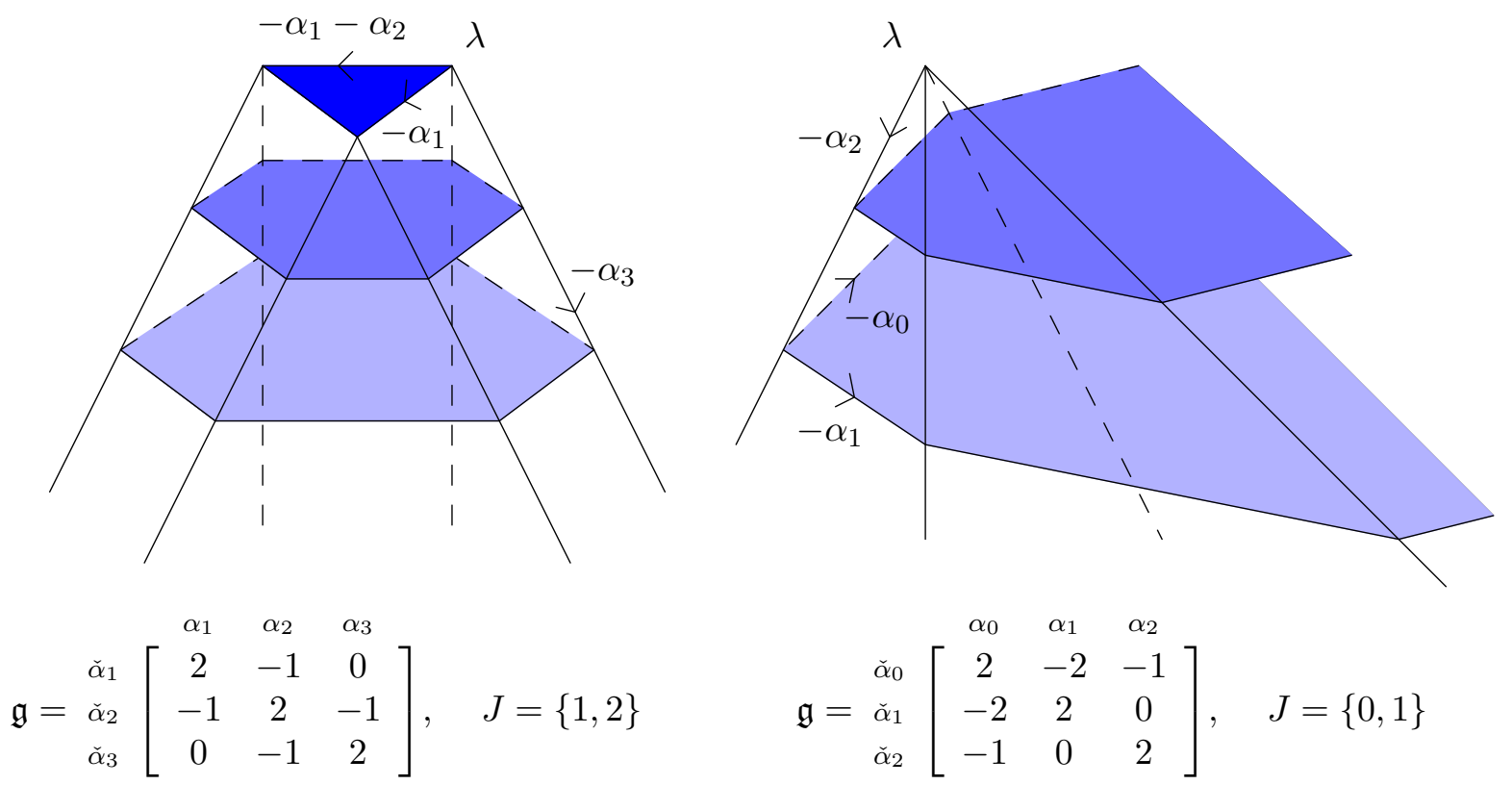

Figure 3.1. Integrable Slice Decomposition, with finite and infinite integrability. Note we only draw four of the infinitely many rays originating at $\lambda$ in the right-hand illustration.

\section{Classifichtion of FaCes of highest Weight modules}

Let $V$ be a $\mathfrak{g}$-module of highest weight $\lambda$. For a standard Levi subalgebra $\mathfrak{l}$, we introduce the notation $\mathrm{wt}_{\mathfrak{l}}:=\operatorname{conv} \mathrm{wt} U(\mathfrak{l}) V_{\lambda}$, and its translates by the integrable Weyl group $w \mathrm{wt}_{\mathrm{l}}, \forall w \in W_{I_{V}}$. We first check these are indeed faces of conv $V$.

Lemma 4.1. $w \mathrm{wt}_{\mathfrak{l}}$ is an (exposed) face of conv $V$, for any standard Levi $\mathfrak{l}$ and $w \in W_{I_{V}}$.

Proof. It suffices to consider $w=1$, i.e., $\mathrm{wt}_{\mathfrak{l}}$. Write $\mathfrak{l}=\mathfrak{l}_{J}$ for some $J \subset I$, and choose 'fundamental coweights' $\breve{\omega}_{i}$ in the real dual of $\mathfrak{h}^{*}$ satisfying $\left(\check{\omega}_{i}, \alpha_{j}\right)=\delta_{i j}, \forall i, j \in I$. Then it is clear that $\sum_{i \in I \backslash J} \check{\omega}_{i}$ is maximized on wt $\mathfrak{l}$, as desired.

The non-trivial assertion is that the converse to Lemma 4.1 also holds, which is the main result of this section:

Theorem 4.2. Every face of conv $V$ is of the form $w \mathrm{wt}_{\mathfrak{l}}$, for some standard Levi $\mathfrak{l}$ and $w \in W_{I_{V}}$.

To prove this, we first examine the orbits of wt $\mathfrak{l}$ under $W_{I_{V}}$, in the spirit of [26]. 

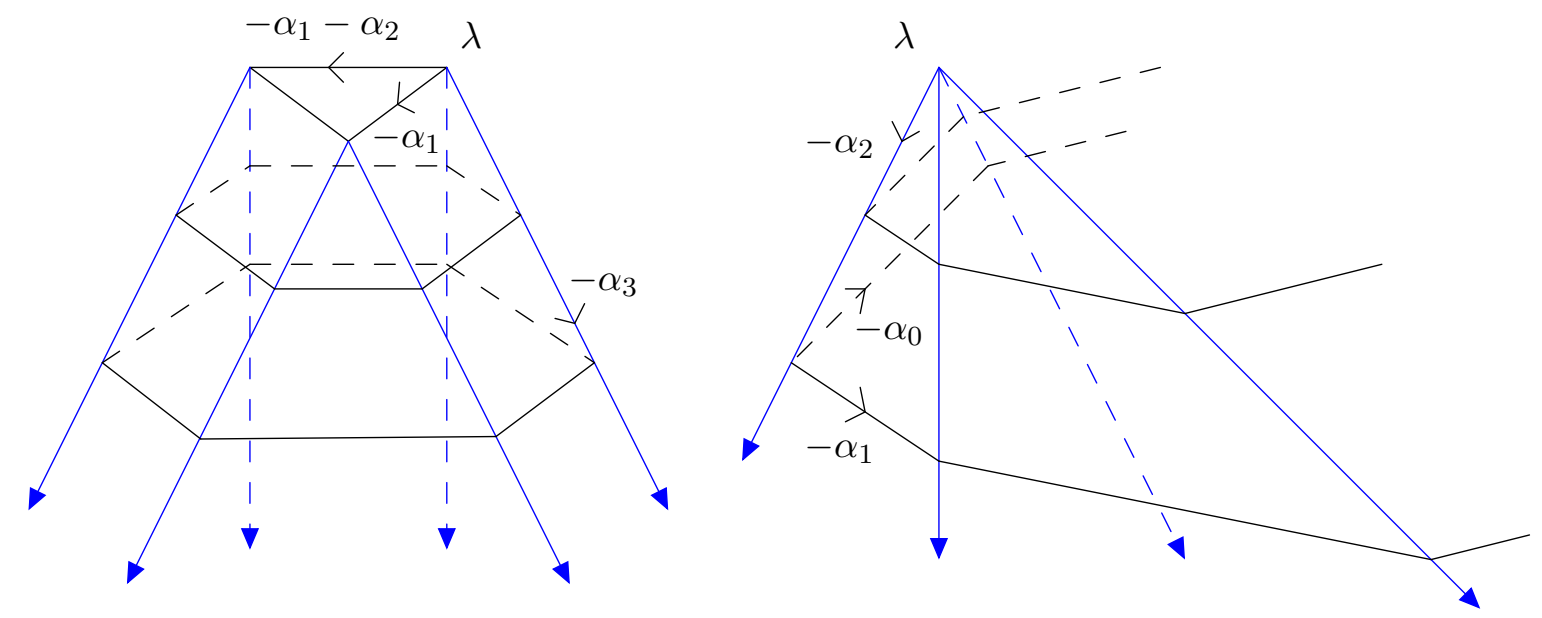

FiguRE 3.2. Ray Decomposition, with finite and infinite integrability; here $\mathfrak{g}$ and $J$ are as in Figure 3.1 .

Proposition 4.3 (Action of $W_{I_{V}}$ on faces). Let $\mathfrak{l}, \mathfrak{l}^{\prime}$ be standard Levi subalgebras, and $w \in W_{I_{V}}$.

(1) $\mathrm{wt}_{\mathfrak{l}}$ has an interior point in the $I_{V}$ dominant chamber.

(2) If $w \mathrm{wt}_{\mathfrak{l}}$ has an interior point in the $I_{V}$ dominant chamber, then $w \mathrm{wt}_{\mathfrak{l}}=\mathrm{wt}_{\mathfrak{l}}$.

(3) If the $W_{I_{V}}$ orbits of $\mathrm{wt}_{\mathfrak{l}}, \mathrm{wt}_{\mathfrak{l}^{\prime}}$ are equal, then $\mathrm{wt}_{\mathfrak{l}}=\mathrm{wt}_{\mathrm{r}^{\prime}}$.

Proof.

(1) Let $\nu$ be an interior point of $\mathrm{wt}_{\mathfrak{l}}$, and write $\mathfrak{l}=\mathfrak{l}_{J}$ for some $J \subset I$. Setting $J^{\prime}=I_{V} \cap J$, it is easy to see $W_{J^{\prime}}$ is the integrable Weyl group of $U\left(\mathfrak{l}_{J}\right) V_{\lambda}$. In particular, by Proposition 3.14, it lies in the $I_{V}$ Tits cone, whence acting by $W_{J^{\prime}}$ we may assume $\nu$ lies in the $J^{\prime}$ dominant chamber, i.e., $\left(\check{\alpha}_{j}, \nu\right) \geqslant 0, \forall j \in$ $J^{\prime}$. Since $\nu$ lies in $\lambda-\mathbb{R}^{\geqslant 0} \pi_{J}$, it follows for all $i \in I_{V} \backslash J$ that $\left(\check{\alpha}_{i}, \nu\right) \geqslant 0$. Thus, $\nu$ lies in the $I_{V}$ dominant chamber.

(2) The proof of (1) showed that in fact any interior point of $\mathrm{wt}_{\mathrm{l}}$ may be brought via $W_{J^{\prime}}$ into the $I_{V}$ dominant chamber. Let $\nu^{\prime}$ be an interior point of $w \mathrm{wt}_{\mathrm{r}}$ which is $I_{V}$ dominant. Then $w^{-1} \nu^{\prime}$ is an interior point of $\mathrm{wt}_{\text {l }}$; pre-composing $w$ with an element of $W_{J^{\prime}}$ we may assume $w^{-1} \nu^{\prime}$ is $I_{V}$ dominant. But since the $I_{V}$ dominant chamber is a fundamental domain for the $I_{V}$ Tits cone (cf. Proposition 3.2), it follows $w^{-1} \nu^{\prime}=\nu^{\prime}$. As the faces $\mathrm{wt}_{\mathfrak{l}}, w \mathrm{wt}_{\mathrm{l}}$ have intersecting interiors, by Lemma 3.10 they coincide.

(3) This is immediate from the first two assertions. 
Proof of Theorem 4.2. We proceed by induction on $|I|$, i.e., the number of simple roots. So we may assume the result for all highest weight modules over all proper Levi subalgebras.

If $F$ is a face of conv $V$, by the Ray Decomposition $3.17 F$ contains $w \lambda$, for some $w \in W_{I_{V}}$. Replacing $F$ by $w^{-1} F$, we may assume $F$ contains $\lambda$. Passing to tangent cones, it follows by Lemma 3.11 that $T_{\lambda} F$ is a face of $T_{\lambda} V$. We observe that the latter has representation theoretic meaning:

Lemma 4.4. Set $J:=I_{V} \cap \lambda^{\perp}$, where $\lambda^{\perp}:=\left\{i \in I_{V}:\left(\check{\alpha}_{i}, \lambda\right)=0\right\}$. Then $T_{\lambda} V=\operatorname{conv} M(\lambda, J)$.

Proof. Composing the surjections $M(\lambda, J) \rightarrow M\left(\lambda, I_{V}\right) \rightarrow V$, we have $T_{\lambda} V \subset$ $T_{\lambda} M(\lambda, J)$. On the other hand, by the Ray Decomposition 3.17 ,

$$
\operatorname{conv} M(\lambda, J)=\operatorname{conv} \bigcup_{w \in W_{J}, i \in I \backslash J} w\left(\lambda-\mathbb{Z}^{\geqslant 0} \alpha_{i}\right)=T_{\lambda} M(\lambda, J),
$$

which evidently lies in $T_{\lambda} V$.

It may be clarifying for the reader in the remainder of the argument to keep in mind the right-hand picture in Figure 3.1 .

We now reduce to proving Theorem 4.2 for $M(\lambda, J)$, with $J=I_{V} \cap \lambda^{\perp}$ as above. Indeed, assuming this holds, by Lemma 3.11 we may write $T_{\lambda} F=w \mathrm{wt}_{\mathfrak{l}} M(\lambda, J)$, for $w \in W_{J}$. Again replacing $F$ with $w^{-1} F$, we may assume $T_{\lambda} F=\mathrm{wt}_{\mathrm{r}} M(\lambda, J)$. By a similar argument to Lemma 4.4. $T_{\lambda} \mathrm{wt}_{\mathrm{l}} V=\mathrm{wt}_{\mathrm{l}} T_{\lambda} V=T_{\lambda} F$. It follows that aff $F=$ aff $\mathrm{wt}_{\mathrm{t}}$, whence $F=\mathrm{wt}_{\mathrm{l}}$ by Lemma 3.9 .

It remains to prove Theorem 4.2 for $V=M(\lambda, J)$. We may assume $J$ is a proper subset of $I$, otherwise $V$ is a 1-dimensional module and the theorem is tautological. Let $F$ be a face of conv $V$. We first claim that $F$ contains a relative interior point $v_{0}$ such that $v_{0} \in \lambda-\mathbb{Q}^{\geqslant 0} \pi$. Indeed, let $V^{\prime}:=\operatorname{conv} V-\lambda, F^{\prime}:=F-\lambda$. Then by the Ray Decomposition 3.17, $V^{\prime}$ is the convex hull of a collection of rational rays $r_{i}=\mathbb{R}^{\geqslant 0} p_{i}, p_{i} \in P_{\mathbb{Q}}$, using the rational structure $P_{\mathbb{Q}} \subset \mathfrak{h}^{*}$ fixed in Section 3.4. If $F^{\prime}$ is not the cone point 0 , for which the theorem is tautological, it is the convex hull of those rays $r_{i}$ whose relative interiors it contains. In particular, $F^{\prime}$ is again a convex hull of rational rays $r_{i}^{\prime}$. It follows that aff $F^{\prime}=P^{\prime} \otimes_{\mathbb{Q}} \mathbb{R}$, where $P^{\prime} \subset P_{\mathbb{Q}}$ is the $\mathbb{Q}$-span of the $r_{i}^{\prime}$. Since relint $F^{\prime}$ is open in aff $F^{\prime}$, it is now clear it contains a rational point, as desired.

We next reduce to the case of an interior point in $\lambda-\mathbb{Z}^{\geqslant 0} \pi$. To see why, note that for any $n \in \mathbb{Z}^{\geqslant 0}$, dilating $\mathfrak{h}^{*}$ by a factor of $n$ gives an isomorphism $d_{n}: \operatorname{conv} M(\lambda, J) \rightarrow \operatorname{conv} M(n \lambda, J)$, e.g. by the Ray Decomposition 3.17. Moreover, $d_{n}$ sends $w \mathrm{wt}_{\mathfrak{l}} M(\lambda, J)$ to $w \mathrm{wt}_{\mathfrak{l}} M(n \lambda, J)$, for all standard Levi subalgebras $\mathfrak{l}$ and $w \in W_{J}$. 
With these reductions, let $v_{0}$ be an interior point of $F$, lying in $\lambda-\mathbb{Z}^{\geqslant 0} \pi$. Since $v_{0}$ lies in the $J$ Tits cone by Proposition 3.14 replacing $F$ by $w F$ for some $w \in W_{J}$, we may assume $v_{0}$ is in the $J$ dominant chamber. Write

$v_{0}=\lambda-\sum_{j \in J^{\prime}} n_{j} \alpha_{j}-\sum_{i \in I^{\prime}} n_{i} \alpha_{i}, \quad$ where $J^{\prime} \subset J, I^{\prime} \subset I \backslash J$, and $n_{j}, n_{i} \in \mathbb{Z}^{>0}, \forall j \in J^{\prime}, i \in I^{\prime}$.

Write $\mathfrak{l}$ for the Levi subalgebra associated to $J^{\prime} \sqcup I^{\prime}$; we claim that $v_{0}$ is also a interior point of $\mathrm{wt}_{\mathfrak{l}}$, which will complete the proof by Lemma 3.10. We address this claim in two steps, by analyzing first the integrable directions $J^{\prime}$, and then incorporating $I^{\prime}$.

Consider the integrable slice conv $L_{\mathfrak{l}_{J}}\left(\lambda^{\prime}\right)$, where $\lambda^{\prime}:=\lambda-\sum_{i \in I^{\prime}} n_{i} \alpha_{i}$. We first claim $v_{0}$ is an interior point of $\mathrm{wt}_{\mathrm{I}_{J^{\prime}}} L_{\mathfrak{l}_{J}}\left(\lambda^{\prime}\right)$. Recalling that $J$ is a proper subset of $I$, the claim follows from the following lemma, applied to the $\mathfrak{l}_{J^{\prime}}$-module generated by $L_{\mathfrak{l}_{J}}\left(\lambda^{\prime}\right)_{\lambda^{\prime}}$.

Lemma 4.5. Assume Theorem 4.2 is true for $\mathfrak{g}$. Suppose a point $v_{0}$ of conv $V$ (i) lies in the $I_{V}$ dominant chamber, and (ii) is of the form $\lambda-\sum_{i \in I} c_{i} \alpha_{i}, c_{i}>0, \forall i \in I$. Then $v_{0}$ is an interior point of conv $V$.

Proof. Iteratively applying the supporting hyperplane theorem, we may assume $v_{0}$ is an interior point of a face $F$. By Theorem 4.2, $F=w w_{\text {l }}$ for some standard Levi subalgebra $\mathfrak{l} \subsetneq \mathfrak{g}$ and $w \in W_{I_{V}}$. By assumption (i) and Proposition 4.3, we may take $w=1$, and now (ii) yields $\mathfrak{l}=\mathfrak{g}$, completing the proof.

Next, it is easy to see that $\operatorname{dim} \operatorname{aff} \mathrm{wt}_{l_{J^{\prime}}} L_{\mathfrak{l}_{J}}\left(\lambda^{\prime}\right)=\left|J^{\prime}\right|$. Indeed, the inequality $\leqslant$ always holds. For the reverse inequality, recall that $v_{0}=\lambda^{\prime}-\sum_{j \in J^{\prime}} n_{j} \alpha_{j}, n_{j}>$ $0, \forall j \in J^{\prime}$. Consider a lowering operator $D$ of weight $-\sum_{j \in J^{\prime}} n_{j} \alpha_{j}$ in $U\left(\mathfrak{n}_{J^{\prime}}^{-}\right)$which does not annihilate the highest weight line of $V=M(\lambda, J)$, and whose existence is guaranteed by Equations (3.15) and (3.16). Using the surjection from the tensor algebra generated by $f_{j}, j \in J^{\prime}$ onto $U\left(\mathfrak{n}_{J^{\prime}}^{-}\right)$, there exists a simple tensor in the $f_{j}$ of weight $-\sum_{j \in J^{\prime}} n_{j} \alpha_{j}$ that does not annihilate the highest weight line. Viewing the simple tensor as a composition of simple lowering operators, it follows that the weight spaces it successively lands in are nonzero. Since $n_{j}>0, \forall j \in J^{\prime}$, this proves the reverse inequality.

Now choose affine independent $p_{0}, \ldots, p_{\left|J^{\prime}\right|}$ in $\mathrm{wt}_{\mathfrak{l}^{\prime}} L_{\mathfrak{l}_{J}}\left(\lambda^{\prime}\right)$ whose convex hull contains $v_{0}$ as an interior point. Write each as a convex combination of $W_{J^{\prime}}\left(\lambda^{\prime}\right)$, i.e.,

$$
p_{k}=\sum_{j} t_{k j} w_{j}^{\prime}\left(\lambda^{\prime}\right), \quad \text { with } \quad w_{j}^{\prime} \in W_{J^{\prime}}, t_{k j} \in \mathbb{R}^{\geqslant 0}, \sum_{j} t_{k j}=1,0 \leqslant k \leqslant\left|J^{\prime}\right| .
$$

As affine independence can be checked by the non-vanishing of a determinant, we may pick a compact neighborhood $B$ around $\sum_{i \in I^{\prime}} n_{i} \alpha_{i}$ in $\mathbb{R}^{>0} \pi_{I^{\prime}}$ such that for 
each $b \in B$, the points $p_{k}(b):=\sum_{j} t_{k j} w_{j}^{\prime}(\lambda-b)$ are again affine independent. Writing $\Sigma(b):=\operatorname{conv}\left\{p_{k}(b): 0 \leqslant k \leqslant\left|J^{\prime}\right|\right\}$, and $\Sigma(B):=\cup_{b \in B} \Sigma(b)$, is it easy to see we have a homeomorphism $\Sigma(B) \simeq \sigma \times B$, where $\sigma$ denotes the standard $\left|J^{\prime}\right|$ simplex. In particular, $v_{0}$ is an interior point of $\Sigma(B)$. Noting that $\Sigma(B) \subset \mathrm{wt}_{\mathrm{l}}$, and dim aff $\mathrm{wt}_{\mathfrak{l}} \leqslant \operatorname{dim}$ aff $\Sigma(B)=\left|J^{\prime}\right|+\left|I^{\prime}\right|$, it follows that dimaff $\Sigma(B)=\operatorname{dim}_{\text {aff }} \mathrm{wt}_{\mathfrak{l}}$, and hence $v_{0}$ is an interior point of $\mathrm{wt}_{\mathrm{l}}$, as desired.

\section{INCLUSIONS BETWEEN FACES}

Having shown in Section 4 that every face is of the form $w \mathrm{wt}_{\mathfrak{l}}$, to complete their classification it remains to address their inclusion relations, and in particular their redundancies. We first ignore the complications introduced by the Weyl group, i.e., classify when $\mathrm{wt}_{\mathfrak{l}} \subset \mathrm{wt}_{\mathfrak{l}^{\prime}}$, for Levi subalgebras $\mathfrak{l}, \mathfrak{l}^{\prime}$.

To do so, we introduce some terminology. For $J \subset I$, call a node $j \in J$ active if either (i) $j \notin I_{V}$, or (ii) $j \in I_{V}$ and $\left(\check{\alpha}_{j}, \lambda\right) \in \mathbb{R}^{>0}$, and otherwise call it inactive. Notice that the active nodes are precisely those $j \in J$ for which $\lambda-\alpha_{j} \in$ wt $V$. Decompose the Dynkin diagram corresponding to $J$ as a disjoint union of connected components $C_{\beta}$, and call a component $C_{\beta}$ active if it contains an active node. Finally, let $J_{\min }$ denote the nodes of all the active components. The above names are justified by the following theorem.

Theorem 5.1. Let $\mathfrak{l}, \mathfrak{l}^{\prime}$ be standard Levi subalgebras, and $J, J^{\prime}$ the corresponding subsets of $I$, respectively. Then $\mathrm{wt}_{\mathfrak{l}} \subset \mathrm{wt}_{\mathfrak{l}^{\prime}}$ if and only if $J_{\min } \subset J^{\prime}$.

Proof. First, by the PBW theorem and $V=U\left(\mathfrak{n}^{-}\right) V_{\lambda}$ :

$$
U(\mathfrak{l}) V_{\lambda}=\bigoplus_{\mu \in \lambda+\mathbb{Z} \pi_{J}} V_{\mu},
$$

so $\mathrm{wt}_{\mathrm{l}}=\operatorname{conv} V \cap\left(\lambda+\mathbb{R} \pi_{J}\right)$. Accordingly, it suffices to check that

$$
\text { aff } \mathrm{wt}_{\mathfrak{l}}=\lambda+\mathbb{R} \pi_{J_{\min }} .
$$

To see $\supset$, for any node $i \in J_{\min }$, we may choose a sequence $i_{1}, \ldots, i_{n}=i$ of distinct nodes in $J_{\min }$ such that (i) $i_{1}$ is active, and (ii) $i_{j}$ is inactive and shares an edge with $i_{j-1}, \forall j \geqslant 2$. Then by basic $\mathfrak{s l}_{2}$ representation theory it is clear the weight spaces corresponding to the following sequence of weights are nonzero:

$$
\lambda-\alpha_{i_{1}}, \quad \lambda-\left(\alpha_{i_{1}}+\alpha_{i_{2}}\right), \quad \ldots, \quad \lambda-\sum_{1 \leqslant j \leqslant n} \alpha_{i_{j}} .
$$

It is now clear that aff $\mathrm{wt}_{\mathfrak{l}}$ contains $\lambda+\mathbb{R} \alpha_{i}$, proving $\supset$ as desired.

For the inclusion $\subset$, by assumption the simple coroots corresponding to $J \backslash J_{\min }$ act by 0 on $\lambda$, and are disconnected from $J_{\min }$ in the Dynkin diagram. In particular, 
as Lie algebras $\mathfrak{n}_{J}^{-}=\mathfrak{n}_{J_{\min }}^{-} \oplus \mathfrak{n}_{J \backslash J_{\min }}^{-}$, whence by the PBW theorem,

$$
U(\mathfrak{l}) V_{\lambda}=U\left(\mathfrak{n}_{J_{\min }}^{-}\right) \otimes_{\mathbb{C}} U\left(\mathfrak{n}_{J \backslash J_{\min }}^{-}\right) V_{\lambda},
$$

and the augmentation ideal of $U\left(\mathfrak{n}_{J \backslash J_{\min }}^{-}\right)$annihilates $V_{\lambda}$, as desired.

Remark 5.5. Equation (5.2) immediately implies a result in [18, namely, that $\mathrm{wt}_{\mathfrak{l}}=\mathrm{wt}_{\mathfrak{l}^{\prime}}$ if and only if $U(\mathfrak{l}) V_{\lambda}=U\left(\mathfrak{l}^{\prime}\right) V_{\lambda}$.

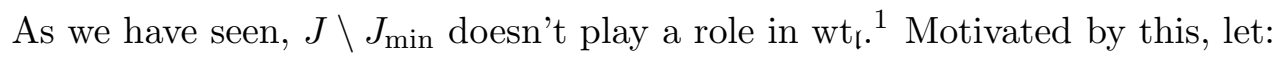

$$
J_{d}:=\left\{j \in I_{V}:\left(\check{\alpha}_{j}, \lambda\right)=0 \text { and } j \text { is not connected to } J_{\min }\right\},
$$

and set $J_{\max }:=J_{\min } \sqcup J_{d}$. Here we think of the subscript $d$ as standing for 'dormant'. An easy adaptation of the argument of Theorem [5.1, in particular Equation (5.3), shows the following:

Theorem 5.7. Let $\mathfrak{l}, \mathfrak{l}^{\prime}$ be standard Levi subalgebras, and $J, J^{\prime}$ the corresponding subsets of $I$. Then $\mathrm{wt}_{\mathfrak{l}}=\mathrm{wt}_{\mathfrak{l}^{\prime}}$ if and only if $J_{\min } \subset J^{\prime} \subset J_{\max }$.

Remark 5.8. We now discuss precursors to Theorem 5.7. The sets $J_{\min }, J \subset I$ appear in Satake [25, §2.3], Borel-Tits [3, §12.16], Vinberg [26, §3], and Casselman [6, §3] for integrable modules in finite type. To our knowledge $J_{\max }$ first appears in work of Cellini-Marietti [7] and subsequent work by Khare [18], again in finite type.

Finally, we comment on the relationship of the language in Theorems 5.1 and 5.7 to the two previous approaches: (i) that of Vinberg [26] and Khare [18], and (ii) that of Satake [25], Borel-Tits [3], Casselman [6], Cellini-Marietti [7], and Li-Cao-Li [21]. Ours is essentially an average of the two different approaches: in our terminology, unlike in [18, we do not distinguish between integrable and non-integrable 'active' nodes, which simplifies the subsets $J_{1}-J_{6}$ of Definition 3.1 of loc. cit. to our $J_{\min }$ and $J_{\max }$. Similarly, if we extend the Dynkin diagram of $\mathfrak{g}$ by adding a node $i_{\star}$ that is connected to exactly the active nodes, then we may rephrase our results along the lines of [3], [6], [7], 221], 25] as follows: standard parabolic faces of conv $V$ are in bijection with connected subsets of the extended Dynkin diagram that contain $i_{\star}$.

Corollary 5.9. Let $L(\lambda)$ be a simple module, and $\mathfrak{l}_{J}$ a standard Levi subalgebra. The restriction of $L(\lambda)$ to $\mathfrak{l}_{J}$ is simple if and only if $I_{\min } \subset J$.

Corollary 5.10. Let $V$ be a highest weight module, and $\mathfrak{l}_{J}$ a standard Levi subalgebra. The restriction of $V$ to $\mathfrak{l}_{J}$ is a highest weight module if and only if $I_{\min } \subset J$.

We are ready to incorporate the action of the integrable Weyl group. Of course, to understand when $w^{\prime} \mathrm{wt}_{\boldsymbol{l}^{\prime}} \subset w \mathrm{wt}_{\mathrm{l}}$, we may assume $w^{\prime}=1$.

\footnotetext{
${ }^{1}$ In fact, an easy adaptation of the proof of Theorem 5.1 shows the derived subalgebra of $\mathfrak{g}_{J \backslash J_{\min }}$ acts trivially on $U(\mathfrak{l}) V_{\lambda}$.
} 
FACES OF HIGHEST WEIGHT MODULES AND THE UNIVERSAL WEYL POLYHEDRON 19

Proposition 5.11. Set $J_{V}^{\max }:=I_{V} \cap J_{\max }$ and $J_{V}^{\min }:=I_{V} \cap J_{\min }$. Given $w \in W_{I_{V}}$, we have $\mathrm{wt}_{\mathrm{r}^{\prime}} \subset w \mathrm{wt}_{\mathrm{r}}$ if and only if (i) $\mathrm{wt}_{\boldsymbol{r}^{\prime}} \subset \mathrm{wt}_{\mathfrak{r}}$ and (ii) the left coset $w W_{J_{V}^{\min }}$ intersects the integrable stabilizer $\mathrm{Stab}_{W_{I_{V}}}\left(\mathrm{wt}_{\mathrm{l}^{\prime}}\right)$ non-emptily.

Proof. One implication is tautological. For the reverse implication, suppose $\mathrm{wt}_{\mathrm{l}^{\prime}} \subset$ $w \mathrm{wt}_{\text {. }}$. Applying Theorem 4.2 to $\mathfrak{g}_{J_{V}^{\min }}$, we have $\mathrm{wt}_{\mathfrak{l}^{\prime}}=w w^{\prime} \mathrm{wt}_{l^{\prime \prime}}$, for some $w^{\prime} \in$ $W_{J_{V}^{\min }}, \mathfrak{l}^{\prime \prime} \subset \mathfrak{l}$. Then by Proposition 4.3, $\mathrm{wt}_{\mathfrak{l}^{\prime}}=\mathrm{wt}_{\mathfrak{l}^{\prime \prime}}=w w^{\prime} \mathrm{wt}_{\mathfrak{l}^{\prime \prime}}$.

Our last main result in this section computes the stabilizers of the parabolic faces of conv $V$, under the action of the integrable Weyl group.

Theorem 5.12. Retaining the notation of Proposition 5.11, we have:

$$
\operatorname{Stab}_{W_{I_{V}}}\left(\mathrm{wt}_{\mathfrak{l}}\right)=W_{J_{V}^{\max }} \simeq W_{J_{V}^{\min }} \times W_{J_{V}^{\max } \backslash J_{V}^{\min }} .
$$

Proof. The inclusion $\supset$ is immediate. For the inclusion $\subset$, write $F$ for the face wt $_{\mathfrak{l}}$, and $D$ for the $I_{V}$ dominant chamber $D_{I_{V}}$. Then by an argument similar to Proposition 4.3, we have that:

$$
F=\bigcup_{w \in W_{J_{V}^{\min }}}(F \cap w D) .
$$

Suppose that $\tilde{w} \in W_{I_{V}}$ stabilizes $F$. Then we have:

$$
F=\bigcup_{w \in W_{J_{V}^{\min }}}(F \cap \tilde{w} w D)
$$

As $F \cap D \cap \tilde{w} w D$ is a face of $F \cap D$ for all $w \in W_{J_{V}^{\min }}$, it follows by dimension

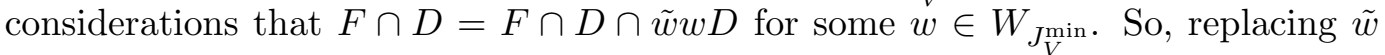
by $\tilde{w} w$, we may assume $\tilde{w}$ fixes $F \cap D$ identically. It suffices to show this implies $\tilde{w} \in W_{J_{d}}$, as defined in Equation (5.6). To see this, for $i \in J_{\min }$, choose as in the proof of Theorem [5.1] a sequence $i_{1}, \ldots, i_{n}=i$ of distinct $i_{j} \in J_{\min }$ such that (i) $i_{1}$ is active, and (ii) $i_{j}$ is inactive and shares an edge with $i_{j-1}, \forall j \geqslant 2$. Write $K(i)=\left\{i_{k}\right\}_{1 \leqslant k \leqslant n}$; it follows from Proposition 4.3(1) that $\mathrm{wt}_{K(i)}$ has an interior point $v_{i}$ in $D$. Write $v_{i}:=\lambda-\sum_{k=1}^{n} c_{k} \alpha_{i_{k}}$, and let $K^{\prime}:=\left\{i_{k}: c_{k}>0\right\}$. Then applying Lemma 4.5 for $\mathfrak{g}_{K^{\prime}}$, it follows that $v_{i}$ is an interior point of $\mathrm{wt}_{K^{\prime}}$, whence $\mathrm{wt}_{K^{\prime}}=\mathrm{wt}_{K(i)}$ by Lemma 3.10. But $K(i)=K(i)_{\min }$, so $K^{\prime}=K(i)$ and we have $c_{k}$ is nonzero, $1 \leqslant k \leqslant n$. Since $\tilde{w}$ fixes $v_{i}$, it follows by Proposition 3.2 that $\tilde{w} \in W_{J(i)}$, where we write:

$$
J(i):=I_{V} \cap v_{i}^{\perp}=\left\{j \in I_{V}:\left(\check{\alpha}_{j}, \lambda-\sum_{k} c_{k} \alpha_{i_{k}}\right)=0\right\} .
$$


Since $i \in J_{\text {min }}$ was arbitrary, by the observation $W_{K^{\prime}} \cap W_{K^{\prime \prime}}=W_{K^{\prime} \cap K^{\prime \prime}}, \forall K^{\prime}, K^{\prime \prime}$ $\subset I$ we have:

$$
\tilde{w} \in W_{K}, \text { where } K:=\left\{k \in I_{V}:\left(\check{\alpha}_{k}, \lambda\right)=0,\left(\check{\alpha}_{k}, \alpha_{i}\right)=0, \forall i \in J_{\min }\right\} .
$$

But this is exactly $J_{d}$, as desired.

We now deduce the coefficients of the $f$-polynomial of the "polyhedral" set conv $V$, although as we presently discuss, conv $V$ is rarely a polyhedron in infinite type. The following result is a consequence of Theorems 4.2 and 5.12, Proposition 4.3, and Equation (5.3).

Proposition 5.14. Write $f_{V}(q)$ for the $f$-polynomial of conv $V$, i.e., $f_{V}(q)=$ $\sum_{i \in \mathbb{Z} \geqslant 0} f_{i} q^{i}$, where $f_{i} \in \mathbb{Z} \geqslant 0 \cup\{\infty\}$ is the number of faces of dimension $i$. Then we have:

$$
f_{V}(q)=\sum_{\mathrm{wt}_{\mathrm{I}_{J}}}\left[W_{I_{V}}: W_{I_{V} \cap J_{\max }}\right] q^{\left|J_{\min }\right|}
$$

where $\mathrm{wt}_{\mathfrak{l}_{J}}$ ranges over the (distinct) standard parabolic faces of $V$ and we allow $f_{V}(q)$ to have possibly infinite coefficients.

Proposition 5.14 was previously proved by Cellini and Marietti [7] for the adjoint representation when $\mathfrak{g}$ is simple, and by the second named author [18] for $\mathfrak{g}$ finitedimensional and most $V$. Thus Proposition 5.14 addresses the remaining cases in finite type, as well as all cases for $\mathfrak{g}$ of infinite type.

Example 5.16. In the special case of $\lambda \in P^{+}$regular, the formula yields:

$$
f_{V}(q)=\sum_{J \subset I}\left[W: W_{J}\right] q^{|J|}
$$

Example 5.18. More generally, if $\lambda$ is $I_{V}$ regular, then writing $V_{\text {top }}$ for the $I_{V}$ integrable slice conv $U\left(\mathfrak{l}_{I_{V}}\right) V_{\lambda}$, we have:

$$
f_{V}(q)=f_{V_{\text {top }}}(q) \cdot(1+q)^{|I|-\left|I_{V}\right|},
$$

where $f_{V_{t o p}}(q)$ is as in (5.17).

Example 5.18 is a combinatorial shadow of the fact that for $\lambda I_{V}$ regular, the standard parabolic faces of conv $V$ are determined by their intersection with the top slice and their extremal rays at $\lambda$ along non-integrable simple roots.

Figure 5.1 shows an example in type $A_{3}$, namely, a permutohedron. In this case the $f$-polynomial equals $q^{3}+14 q^{2}+36 q+24$, and on facets we get $14=4+4+6$, corresponding to four hexagons each from $\{1,2\}$ and from $\{2,3\}$, and six squares from $\{1,3\}$. 
FACES OF HIGHEST WEIGHT MODULES AND THE UNIVERSAL WEYL POLYHEDRON 21

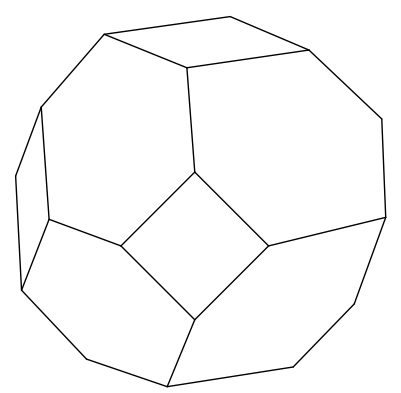

Figure 5.1. $A_{3}$-permutohedron (i.e., $\lambda \in P^{+}$regular)

\section{FAmilies of Weyl Polyhedra AND Representability}

Let $\mathfrak{g}$ be a Kac-Moody algebra, and $\mathfrak{p}$ a parabolic subalgebra with Levi quotient $\mathfrak{l}$. Consider ind $\mathfrak{\mathfrak { p }}_{\mathfrak{p}}^{\mathfrak{g}} L$, for $L$ an integrable highest weight $\mathfrak{l}$ module. This induced module still has a weight decomposition with respect to a fixed Cartan of $\mathfrak{l}$, and one can study the convex hulls of the weights of these modules as $L$ varies.

Less invariantly, if $\mathfrak{l}$ corresponds to a subset $J \subset I$ of the simple roots, the resulting convex hulls are precisely those of the parabolic Verma modules with integrability $J$, or equivalently of all highest weight modules with integrability $J$.

We now introduce a family of convex shapes interpolating between the above convex hulls. In particular, for the remainder of this section we fix a subset of the simple roots $J \subset I$.

Definition 6.1. Say a subset $P \subset \mathfrak{h}^{*}$ is a Weyl polyhedron if it can be written as a convex combination:

$$
P=\sum_{i} t_{i} \operatorname{conv} V_{i}, \quad t_{i}>0, \sum_{i} t_{i}=1
$$

where $V_{i}$ are highest weight modules with integrability $J$.

We will see shortly that Weyl polyhedra are always polyhedra if and only if the corresponding Levi is finite dimensional, so this is strictly speaking abuse of notation.

Having introduced 'real analogues' of convex hulls of parabolically induced modules, we can now let convex hulls vary in families. By a convex set $S$, we simply mean a subset of some real vector space which is convex is the usual sense.

Definition 6.2. Let $S$ be a convex set. Define an $S$ family of Weyl polyhedra to be a subset $\mathcal{P} \subset S \times \mathfrak{h}^{*}$ satisfying:

(1) Under the natural projection $\pi: \mathcal{P} \rightarrow S$, the fibers $F_{s}:=\pi^{-1} s$ are Weyl polyhedra for all $s \in S$; 
(2) For $s_{0}, s_{1} \in S, 0 \leqslant t \leqslant 1$, we have:

$$
t F_{s_{0}}+(1-t) F_{s_{1}}=F_{t s_{0}+(1-t) s_{1}} .
$$

We remind that a morphism of convex sets $S \rightarrow S^{\prime}$ is a map of sets which commutes with taking convex combinations. With this, we have that families of Weyl polyhedra pull back.

Lemma 6.3. If $\phi: S^{\prime} \rightarrow S$ is a morphism of convex sets, and $\mathcal{P} \rightarrow S$ is an $S$ family of Weyl polyhedra, then the base change $\phi^{*} \mathcal{P}:=\mathcal{P} \times_{S} S^{\prime}$ is an $S^{\prime}$ family of Weyl polyhedra.

It follows that the assignment $\mathrm{F}: S \rightsquigarrow\{S$ families of Weyl polyhedra $\}, \phi \rightsquigarrow$ $\phi^{*}$, defines a contravariant functor from convex sets to sets. We now construct what could be loosely thought of as a convexity-theoretic Hilbert scheme for Weyl polyhedra.

Theorem 6.4. The functor $\mathrm{F}$ is representable.

From the theorem, we obtain a unique up to unique isomorphism base space $B$ and universal family $\mathcal{P}_{\text {univ }} \rightarrow B$. In the subsequent section, we will show that the total space of $\mathcal{P}_{\text {univ }}$ is a remarkable convex cone, whose combinatorial isomorphism type stores the classification of faces obtained in the previous sections.

Proof. We need to show there exists a convex set $B$ and bijections for every convex set $S$ :

$$
\{S \text { families of Weyl polyhedra }\} \simeq \operatorname{Hom}(S, B)
$$

which intertwine pullback of families and precomposition along morphisms $S \rightarrow S^{\prime}$.

To do so, we introduce in the following lemma a Ray Decomposition for Weyl polyhedra, akin to Proposition 3.17

Lemma 6.6. For $\lambda \in D_{J}$, i.e. $\left(\check{\alpha}_{j}, \lambda\right) \in \mathbb{R}^{\geqslant 0}, \forall j \in J$, set:

$$
P(\lambda, J):=\mathrm{conv} \bigcup_{w \in W_{J}, i \in I \backslash J} w\left(\lambda-\mathbb{R}^{\geqslant 0} \alpha_{i}\right) .
$$

If $J=I$, by the RHS we mean conv $\bigcup_{w \in W} w \lambda$. Then for any finite collection $\lambda_{i} \in D_{J}, t_{i} \geqslant 0, \sum_{i} t_{i}=1$, we have:

$$
\sum_{i} t_{i} P\left(\lambda_{i}, J\right)=P\left(\sum_{i} t_{i} \lambda_{i}, J\right)
$$

The lemma is proved by direct calculation.

Notice that a highest weight module $V$ with integrability $J$ and highest weight $\lambda$ has conv $V=P(\lambda, J)$. It follows from Equation (6.8) that Weyl polyhedra are precisely the shapes $P(\lambda, J)$ for $\lambda \in D_{J}$. As these have a unique vertex in the $J$ 
dominant chamber, namely $\lambda$, they in particular are distinct, and thus we have a naive parametrization of Weyl polyhedra by their highest weights.

Introduce the tautological family $\mathcal{P}_{J} \rightarrow D_{J}$, where we define:

$$
\mathcal{P}_{J}:=\left\{(\lambda, \nu): \lambda \in D_{J}, \nu \in P(\lambda, J)\right\} .
$$

A direct calculation shows that $\mathcal{P}_{J}$ is a $D_{J}$ family of Weyl polyhedra.

We now show that $D_{J}$ is the moduli space that represents $\mathrm{F}$, with universal family $\mathcal{P}_{\text {univ }}=\mathcal{P}_{J}$. Indeed, given a morphism of convex sets $\phi: S \rightarrow D_{J}$, we associate to it the pullback of the tautological family $\phi^{*} \mathcal{P}_{J}$. In the reverse direction, given an $S$ family of Weyl polyhedra, if the fiber over $s \in S$ is $P(\lambda(s), J)$, then the map $s \mapsto \lambda(s)$ is a morphism of convex sets by Equation (6.8). One can directly verify these assignments are mutually inverse, and intertwine precomposition and pullback of families under a convex morphism $S \rightarrow S^{\prime}$.

Remark 6.10. Notice that both sides of Equation (6.5) are naturally convex sets (given by taking convex combinations fiberwise and pointwise, respectively), and in fact the isomorphism is one of convex sets.

Example 6.11. For a simplex $S$ with vertices $v_{0}, \ldots, v_{n}$, an $S$ family of Weyl polyhedra is the same datum as the fibers at the vertices, i.e. Weyl polyhedra $P_{0}, \ldots, P_{n}$. Thus in this case, both sides of Equation (6.5) indeed reduce to $D_{J}^{n+1}$.

Example 6.12. We could just as well have parametrized Weyl polyhedra by their unique vertex in another fixed chamber of the $J$ Tits cone. Writing the chamber as $w D_{J}, w \in W_{J}$, the isomorphism of convex sets $w D_{J} \simeq D_{J}$ corresponding to Theorem 6.4 is simply application of $w^{-1}$.

We conclude this section with an illustration of a universal family of Weyl polyhedra; see Figure 6.1.

\section{FACES OF the Universal Weyl POLYHEDRON}

In the previous section, we began studying families of Weyl polyhedra $\mathcal{P} \rightarrow S$. In this section, we study faces of such families, i.e. of the total spaces $\mathcal{P}$, which indeed are convex. Notice that just as families pull back, so do faces:

Lemma 7.1. Let $\phi: S^{\prime} \rightarrow S$ be a morphism of convex sets, $\mathcal{P} \rightarrow S$ a family of Weyl polyhedra, and $F \rightarrow \mathcal{P}$ a face of the total space. Then the pullback $\phi^{*} F$ is a face of $\phi^{*} \mathcal{P}$.

We motivate what follows with a very natural face of any family. As before, we work with Weyl polyhedra corresponding to a fixed $J \subset I$. 


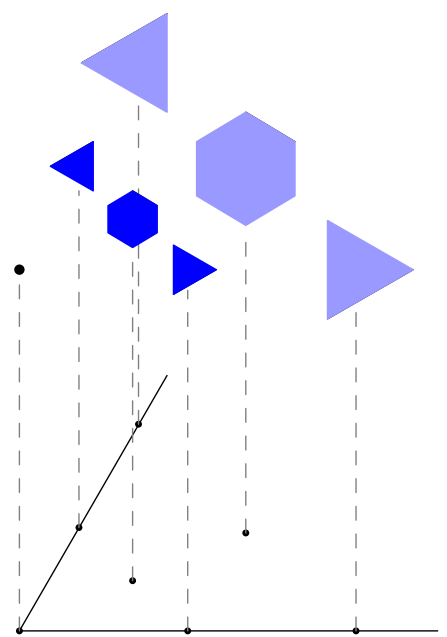

FiguRE 6.1. Schematic of the universal family of Weyl polyhedra for $\mathfrak{g}=\mathfrak{s l}_{3}$ and $J=I$. The fibers $P(\lambda, J)$ are illustrated at some points $\lambda$ along the relative interiors of each face in the dominant chamber $D=D_{I}$.

Example 7.2. For $j \in J$, consider the corresponding root hyperplane $H_{j}$ in $\mathfrak{h}^{*}$. If $\mathcal{P} \rightarrow S$ is a family of Weyl polyhedra, then the union of the fibers whose highest weight lies in $H_{j}$ is a face of $\mathcal{P}$.

Notice in the above example that this construction commutes with pullback of families. In this way, one can study 'characteristic faces', i.e. the assignment to every family of Weyl polyhedra $\mathcal{P}$ of a face $F \subset \mathcal{P}$ which commutes with pullback. As the intersection of each face with a given fiber is a face of the fiber, one may think of characteristic faces as controlling how the faces of conv $V$ deform as one deforms the highest weight.

As families of Weyl polyhedra admit a moduli space, i.e. Theorem 6.4, characteristic faces are the same data as faces of $\mathcal{P}_{\text {univ }}$, which we call the universal Weyl polyhedron. In this section, we will determine $\mathcal{P}_{\text {univ }}$ up to combinatorial isomorphism, i.e. enumerate all faces as well as their dimensions and inclusions.

The main ingredients will be (i) an extension of the classification of faces of conv $V$ from Sections 4, 5 to all Weyl polyhedra, and (ii) a stratification of the base $D_{J}$ along which $\mathcal{P}_{\text {univ }}$ is 'constant'. The latter will in particular strengthen the consequence of Section 5 that the classification of faces of conv $V$ only depends on the singularity of the highest weight, which followed from the definitions of $J_{\min }, J_{\max }$. 
7.1. Faces of Weyl polyhedra. In this subsection, we show the classification of faces of $P(\lambda, J)$. It is exactly parallel to that of $\operatorname{conv} M(\lambda, J)$, and we elaborate whenever the proofs differ.

For $J^{\prime} \subset I$, with associated Levi subalgebra $\mathfrak{l}$, consider the functional $\sum_{i \in I \backslash J^{\prime}} \check{\omega}_{i}$ as in Lemma 4.1] As $P(\lambda, J) \subset \lambda-\mathbb{R}^{\geqslant 0} \pi, \sum_{i \in I \backslash J^{\prime}} \check{\omega}_{i}$ attains a maximum on $P(\lambda, J)$, and write $\mathrm{wt}_{\mathfrak{l}}$ for the corresponding exposed face. As $P(\lambda, J)$ is $W_{J}$ invariant, similarly $w \mathrm{wt}_{\mathfrak{l}}$ is a face of $P(\lambda, J)$, for $w \in W_{J}$.

Theorem 7.3. Every face of $P(\lambda, J)$ is of the form $w \mathrm{wt}_{\mathfrak{l}}$, for $w \in W_{J}$ and a standard Levi algebra $\mathfrak{l}$.

Proof. This is proved similarly to Theorem 4.2. The analogue of Lemma 4.4 may be proved as follows.

Lemma 7.4. $T_{\lambda} P(\lambda, J)=P\left(\lambda, J \cap \lambda^{\perp}\right)$, where $\lambda^{\perp}:=\left\{i \in I:\left(\check{\alpha}_{i}, \lambda\right)=0\right\}$.

Proof. The inclusion $\supseteq$ is as in Lemma 4.4. For the inclusion $\subseteq$, we prove by induction on $\ell(w)$ that $w\left(\lambda-t \alpha_{i}\right) \in P\left(\lambda, J \cap \lambda^{\perp}\right)$, for $t \geqslant 0, i \notin J, w \in W_{J}$. The base case of $w=1$ is clear. For the induction step write $w=s_{j} \tilde{w}$, with $\ell(\tilde{w})<\ell(w), j \in J$. If $j \in \lambda^{\perp}$, we use the inductive hypothesis for $\tilde{w}$ and the $s_{j}$ invariance of $P\left(\lambda, J \cap \lambda^{\perp}\right)$. If $j \notin \lambda^{\perp}$, since $\lambda$ is $J$-dominant, we have:

$$
w\left(\lambda-t \alpha_{i}\right)=\tilde{w}\left(\lambda-t \alpha_{i}\right)-t^{\prime} \alpha_{j}, \quad t^{\prime} \in \mathbb{R}^{>0} .
$$

The proof concludes by noting that $P\left(\lambda, J \cap \lambda^{\perp}\right)$ is a cone with vertex $\lambda$ and contains $\lambda-\mathbb{R}^{\geqslant 0} \alpha_{j}$.

Following the notation of the proof of Theorem 4.2, the only argument which requires nontrivial modification is the equality $\operatorname{dimaff} \mathrm{wt}_{J_{J^{\prime}}} L_{\mathfrak{l}_{J}}\left(\lambda^{\prime}\right)=\left|J^{\prime}\right|$. This however is a consequence of the following Theorem 7.5.

We next determine the inclusions between standard parabolic faces. For $J^{\prime} \subset I$, the notions of active and inactive nodes as well as $J_{\min }^{\prime}, J_{\max }^{\prime}$ are as in Section 5 , provided one replaces $I_{V}$ with $J$ in the definitions. With this, we have:

Theorem 7.5. Let $\mathfrak{l}^{\prime}, \mathfrak{l}^{\prime \prime}$ be standard Levi subalgebras, and $J^{\prime}, J^{\prime \prime}$ the corresponding subsets of $I$, respectively. Then $\mathrm{wt}_{\mathrm{l}^{\prime}} \subset \mathrm{wt}_{\mathrm{l}^{\prime \prime}}$ if and only if $J_{\min }^{\prime} \subset J^{\prime \prime}$.

Proof. It suffices to check that aff $\mathrm{wt}_{\mathrm{l}^{\prime}}=\lambda+\mathbb{R} \pi_{J_{\min }^{\prime}}$. The inclusion $\supset$ is proved as in Theorem 5.1, replacing $\lambda-\alpha_{i_{1}}$ with $\lambda-\epsilon_{1} \alpha_{i_{1}}$ for some $\epsilon_{1}>0, \lambda-\alpha_{i_{1}}-\alpha_{i_{2}}$ with $\lambda-\epsilon_{1} \alpha_{i_{1}}-\epsilon_{2} \alpha_{i_{2}}$, for some $\epsilon_{2}>0$, and so on. For the inclusion $\subset$, we will show a 'Ray Decomposition' for each face:

$$
\mathrm{wt}_{\boldsymbol{l}^{\prime}}=\operatorname{conv} \underset{w \in W_{J \cap J^{\prime}}, i \in J^{\prime} \backslash J}{\bigcup} w\left(\lambda-\mathbb{R}^{\geqslant 0} \alpha_{i}\right) .
$$


Having shown this, we will be done, as we may factor $W_{J \cap J^{\prime}} \simeq W_{J \cap J_{\min }^{\prime}} \times W_{J^{\prime} \backslash J_{\min }^{\prime}}$, and the latter parabolic factor acts trivially on $\lambda-\mathbb{R}^{\geqslant 0} \alpha_{i}, \forall i \in J^{\prime} \backslash J$. To see Equation (7.6), the inclusion $\supset$ is straightforward. For the inclusion $\subset$, it suffices to see that if $w\left(\lambda-t \alpha_{i}\right)$ lies in $\mathrm{wt}_{\mathrm{f}^{\prime}}$, for some $w \in W_{J}, t \geqslant 0$, and $i \in I \backslash J$, then (i) $i \in J^{\prime} \backslash J$, and (ii) $w\left(\lambda-t \alpha_{i}\right)=\tilde{w}\left(\lambda-t \alpha_{i}\right)$, for some $\tilde{w}$ in $W_{J \cap J^{\prime}}$. But (i) follows from the linear independence of simple roots, and (ii) follows from noting that $w\left(\lambda-t \alpha_{i}\right)$ lies in the $J \cap J^{\prime}$ Tits cone, and $\lambda-t \alpha_{i}$ lies in the $J$ dominant chamber, which is a fundamental domain for the action of $W_{J}$, cf. Proposition 3.2(2).

The remaining results of Section 5, namely Theorem 5.7. Proposition 5.11, Theorem 5.12, and Proposition 5.14 hold for $P(\lambda, J)$ mutatis mutandis, with similar proofs. In particular, the face poset of $P(\lambda, J)$ depends on $\lambda \in D_{J}$ only through $J \cap \lambda^{\perp}$, proving Theorem 2.4(2).

7.2. Strong combinatorial isomorphism and strata of the Weyl chamber. Before continuing, let us sharpen our observation above that the combinatorial isomorphism class of $P(\lambda, J)$ depends only on the 'integrable stabilizer' of $\lambda$. To do so, we first remind the face structure of $D_{J}$ :

Lemma 7.7. Faces of $D_{J}$ are in bijection with subsets of $J$, under the assignment:

$$
K \subset J \rightsquigarrow{ }^{\perp} K:=\left\{\lambda \in D_{J}:\left(\check{\alpha}_{k}, \lambda\right)=0, \forall k \in K\right\} .
$$

Moreover, this bijection is an isomorphism of posets, where we order faces by inclusion and subsets of $J$ by reverse inclusion.

Let us call the interior of a face of $D_{J}$ a stratum of $D_{J}$. With this, our earlier observation is that the combinatorial isomorphism class of $P(\lambda, J)$ is constant on strata of $D_{J}$.

To explain the sharpening, we recall a notion from convexity, cf. [1. Two convex sets $C, C^{\prime}$ in a real vector space $E$ are said to be strongly combinatorially isomorphic if for all functionals $\phi \in E^{*}$, we have (i): $\phi$ attains a maximum value on $C$ if and only if $\phi$ attains a maximum value on $C^{\prime}$, and (ii) if the maxima exist, writing $F, F^{\prime}$ for the corresponding exposed faces of $C, C^{\prime}$, we have $\operatorname{dim} F=\operatorname{dim} F^{\prime}$. Informally, strong combinatorial isomorphism is combinatorial isomorphism plus 'compatible' embeddings in a vector space.

Proposition 7.8. Fix $\lambda, \lambda^{\prime} \in D_{J}$. Then:

(1) If $P(\lambda, J)$ and $P\left(\lambda^{\prime}, J\right)$ are strongly combinatorially isomorphic, then $\lambda$ and $\lambda^{\prime}$ lie in the same stratum of $D_{J}$.

(2) If $\lambda, \lambda^{\prime}$ have finite stabilizer in $W_{J}$, then the converse to (1) holds, i.e. if they lie in the same stratum $D_{J}$, then $P(\lambda, J)$ and $P\left(\lambda^{\prime}, J\right)$ are strongly combinatorially isomorphic. 
Proof. To see (1), if $P(\lambda, J)$ and $P\left(\lambda^{\prime}, J\right)$ are strongly combinatorially isomorphic, using the functionals $\sum_{i \neq j} \check{\omega}_{i}, j \in J$ shows that $\lambda, \lambda^{\prime}$ lie in the interior of the same face of $D_{J}$. To see (2), let $\phi$ be a functional which is bounded on $P(\lambda, J)$. By applying some $w \in W_{J}$ to $\phi$, we may assume $F_{\phi}$ contains $\lambda$. By the assumption of finite stabilizer in $W_{J}$, we may further assume $\left(\phi, \alpha_{j}\right) \geqslant 0$ for all $j \in J \cap \lambda^{\perp}$. Since $\lambda-\epsilon \alpha_{i} \in P(\lambda, J)$ for some $\epsilon>0, i \notin J \cap \lambda^{\perp}$, it follows for $P(\lambda, J), P\left(\lambda^{\prime}, J\right)$ that $\phi$ cuts out the standard parabolic face corresponding to $\left\{i \in I:\left(\phi, \alpha_{i}\right)\right\}=0$. As by assumption $\lambda^{\prime}, \lambda$ lie in the same faces of the $J$ dominant chamber, the equality of dimensions follows from Theorem 7.5 .

Remark 7.9. It would be interesting to know if Proposition $7.8(2)$ holds without the assumption of finite integrable stabilizer. Note also that the proof contains a short argument for the classification of faces for $P(\lambda, J)$ when $\lambda$ has finite integrable stabilizer.

7.3. Faces of the universal Weyl polyhedron. We now determine the face poset of $\mathcal{P}_{\text {univ }}=\mathcal{P}_{J}$. More generally, in this subsection we do so for $\mathcal{P} \rightarrow S$ an arbitrary family of Weyl polyhedra, where $S$ admits a convex embedding into a finite dimensional real vector space.

Write $\mathcal{F}$ for the face poset of $S$. By our assumption on $S$, it is stratified by the relative interiors of its faces:

$$
S=\bigsqcup_{F \in \mathcal{F}} S_{F}
$$

We first note that with respect to this stratification, $\mathcal{P}$ is 'constant' on strata:

Lemma 7.10. Let $F \in \mathcal{F}$. Then for all $s, s^{\prime} \in S_{F}, \lambda(s)^{\perp}=\lambda\left(s^{\prime}\right)^{\perp}$, where as before $\lambda(s)$ denotes the highest weight of the fiber over $s$.

In particular, the fibers over $S_{F}$ are combinatorially isomorphic.

Proof. Under the corresponding convex morphism $F \rightarrow D_{J}$, if an interior point of $F$ lands in the interior of a face of $D_{J}$, then the interior of $F$ lands in the interior of that face.

We now produce 'tautological' faces of $\mathcal{P}$ by taking families of standard parabolic faces along the closure of a stratum.

Proposition 7.11. For $F \in \mathcal{F}$, and a standard Levi $\mathfrak{l}$ of $\mathfrak{g}$, the locus:

$$
\mathrm{wt}_{\mathfrak{l}, F}:=\left\{(s, x): s \in F, x \in \mathrm{wt}_{\mathfrak{l}} P(\lambda(s), J)\right\}
$$

is a face of $\mathcal{P}$.

Proof. The pullback of the face $F$ along the projection $\pi: \mathcal{P} \rightarrow S$ yields a face $\pi^{*} F$ of $\mathcal{P}$. As in Lemma 4.1, consider the exposed face of $\pi^{*} F$ corresponding to the 
vanishing locus of $(s, x) \mapsto \sum_{i \notin J}\left(\check{\omega}_{i}, \lambda(s)-x\right)$, to obtain the desired face $\mathrm{wt}_{\mathrm{l}, F}$ of $\mathcal{P}$.

Notice that $W_{J}$ acts on $\mathcal{P}$ via the usual action on the second factor. Thus we obtain more faces $w \mathrm{wt}_{\mathrm{r}, F}$ of $\mathcal{P}$ - in fact, all of them:

Theorem 7.12. Every face of $\mathcal{P}$ is of the form $w \mathrm{wt}_{\mathfrak{l}, F}$, for some $w \in W_{J}$, standard Levi $\mathfrak{l}$, and $F \in \mathcal{F}$.

Proof. For any face of $\mathcal{P}$, choose an interior point $(s, x), s \in S, x \in P(\lambda(s), J)$. By acting by $W_{J}$, we may assume that $x$ is $J$ dominant. By Theorem 7.3 and the analogue of Proposition 4.3, $x$ is an interior point of $\mathrm{wt}_{\mathfrak{l}} P(\lambda(s), J)$ for a standard Levi l. For some $F \in \mathcal{F}, s \in S_{F}$. As in the proof of Theorem 4.2, we produce a "product" neighborhood of $(s, x)$ in $\mathrm{wt}_{\mathrm{l}, F}$ in the following manner. Take a relatively open simplex in $\mathrm{wt}_{\mathfrak{l}} P(\lambda(s), J)$ containing $x$. Writing its vertices as convex combinations of the terms in the Ray Decomposition (7.6), in an open neighborhood of $s$ in $S_{F}$, the corresponding convex combinations are affine independent. By dimension counting, $(s, x)$ is an interior point of $\mathrm{wt}_{\mathrm{l}, F}$, and we are done by Lemma 3.10.

Remark 7.13. Theorem 7.12 shows that every face of an $S$ family of Weyl polyhedra is again a family of Weyl polyhedra, provided one passes to (i) a face of the base, and to (ii) a Levi subalgebra of $\mathfrak{g}$.

We next deduce the structure of the face poset of $\mathcal{P}$ from our prior analysis of the face posets of the fibers.

Proposition 7.14. Let $J^{\prime}, J^{\prime \prime} \subset I$, with associated Levi subalgebras $\mathfrak{l}^{\prime}, \mathfrak{l}^{\prime \prime}$, and let $F^{\prime}, F^{\prime \prime} \in \mathcal{F}$. Below, we calculate $J_{\min }^{\prime}$ and $J_{\max }^{\prime}$ for $s$ lying in the stratum of $S$ corresponding to $F^{\prime}$, cf. Lemma 7.10. Then:

(1) $\mathrm{wt}_{\mathrm{l}^{\prime}, F^{\prime}} \subset \mathrm{wt}_{\mathrm{l}^{\prime \prime}, F^{\prime \prime}}$ if and only if (i) $F^{\prime} \subset F^{\prime \prime}$ and (ii) $J_{\min }^{\prime} \subset J^{\prime \prime}$.

(2) $\mathrm{wt}_{l^{\prime}, F^{\prime}}=\mathrm{wt}_{l^{\prime \prime}, F^{\prime \prime}}$ if and only if (i) $F^{\prime}=F^{\prime \prime}$ and (ii) $J_{\min }^{\prime} \subset J^{\prime \prime} \subset J_{\max }^{\prime}$.

(3) For $w \in W_{J}$, we have $w \mathrm{wt}_{\mathrm{l}^{\prime}, F^{\prime}} \subset \mathrm{wt}_{\mathrm{l}^{\prime \prime}, F^{\prime \prime}}$ if and only if (i) $F^{\prime} \subset F^{\prime \prime}$, and (ii) for an interior point $s$ of $F^{\prime}$, we have $w \mathrm{wt}_{\mathrm{l}^{\prime}} P(\lambda(s), J) \subset \mathrm{wt}_{\mathrm{l}^{\prime \prime}} P(\lambda(s), J)$, cf. Proposition 5.11.

(4) The stabilizer of $\mathrm{wt}_{\boldsymbol{l}^{\prime}, F^{\prime}}$ in $W_{J}$ is $W_{J_{\max }^{\prime} \cap J}$.

In particular, we obtain the following formula for the $f$-polynomial of $\mathcal{P}$ :

Proposition 7.15. For $F \in \mathcal{F}$, write $f_{F}(q)$ for the $f$ polynomial of $P(\lambda(s), J), s \in$ $S_{F}$. Then:

$$
f_{\mathcal{P}}(q)=\sum_{F \in \mathcal{F}} q^{\operatorname{dim} F} f_{F}(q)
$$

The above results may be specialized to the case of the universal Weyl polyhedron $\mathcal{P}_{\text {univ }}=\mathcal{P}_{J}$ using the description of the face poset of the base $D_{J}$ reminded in Lemma 
7.7. Before doing so, we first determine when the universal Weyl polyhedron is indeed polyhedral.

Proposition 7.16. $\mathcal{P}_{J}$ is a polyhedron if and only if $J$ is of finite type.

Remark 7.17. In fact, one can show that if $J$ is of finite type, then for any family $\mathcal{P} \rightarrow S, \mathcal{P}$ is a polyhedron if and only if $S$ is.

Proof of Proposition 7.16. If $J$ is not of finite type, consider the tautological faces over the open stratum of $D_{J}$. If $J$ is of finite type, the polyhedrality of $\mathcal{P}_{J}$ follows from the following observation.

Proposition 7.18. For $J \subset I$ arbitrary, $\mathcal{P}_{J}$ is the Minkowski sum of the following rays and lines:

$$
\mathbb{R}^{\geqslant 0}\left(0,-w \alpha_{i}\right), \quad \mathbb{R}^{\geqslant 0}\left(\omega_{j}, w \omega_{j}\right), \quad \mathbb{R}\left(\nu_{k}, \nu_{k}\right),
$$

where $i \in I \backslash J, w \in W_{J}, j \in J$, the $\omega_{j}$ satisfy: $\left(\check{\alpha}_{j^{\prime}}, \omega_{j}\right)=\delta_{j, j^{\prime}}, \forall j, j^{\prime} \in J$, and $\nu_{k}$ form a basis of $\bigcap_{j \in J} \check{\alpha}_{j}^{\perp}$.

Returning to the specialization of the results about a general family $\mathcal{P} \rightarrow S$, the $f$-polynomial of the universal Weyl polyhedron is given by:

Proposition 7.19. Recalling our convention for $D_{J}$, cf. Remark 3.1, we have:

$$
f_{\mathcal{P}_{J}}(q)=q^{2 \operatorname{dim}_{\mathbb{C}} \mathfrak{h}^{*}-\mathrm{rkg}} \sum_{K \subset J} q^{-K} f_{P\left(\lambda_{K}, J\right)}(q),
$$

where $\lambda_{K}$ is any point in the interior of the face ${ }^{\perp} K$ of $D_{J}$.

Remark 7.21. Under the normal convention that the $J$ dominant chamber $D_{J}$ lies in a real form of $\mathfrak{h}^{*}$, Equation (17.20) applies provided one replaces the leading exponent with $\operatorname{dim}_{\mathbb{C}} \mathfrak{h}^{*}$.

Example 7.22. Figure 7.1 shows the two universal Weyl polyhedra for $\mathfrak{g}=\mathfrak{s l}_{2}$. In particular, $f_{\mathcal{P}_{I}}(q)=(1+q)^{2}$ for the first polyhedron. For the second, i.e. the case of empty integrability $J=\emptyset$, Proposition 7.15 implies more generally for any $\mathfrak{g}$ that:

$$
f_{\mathcal{P}_{\emptyset}}(q)=q^{2 \operatorname{dim}_{\mathbb{C}} \mathfrak{h}^{*}-\mathrm{rkg}}(1+q)^{\mathrm{rkg}} .
$$

Of course, strata of the base $D_{J}$ parametrize classes of highest weights that are 'equisingular'. Equation (7.20) is a numerical shadow of the fact that in the classification of faces of $\mathcal{P}_{\text {univ }}$, there is a contribution from the combinatorial isomorphism type of each class of equisingular highest weights exactly once. Thus, the classification of faces for $\mathcal{P}_{\text {univ }}$ is equivalent to the classification of faces of all highest weight modules with integrability $J$. Moreover, the combinatorial isomorphism type of $\mathcal{P}_{\text {univ }}$, i.e. inclusions of faces, stores the data of how parabolic faces degenerate as one specializes the highest weight. 

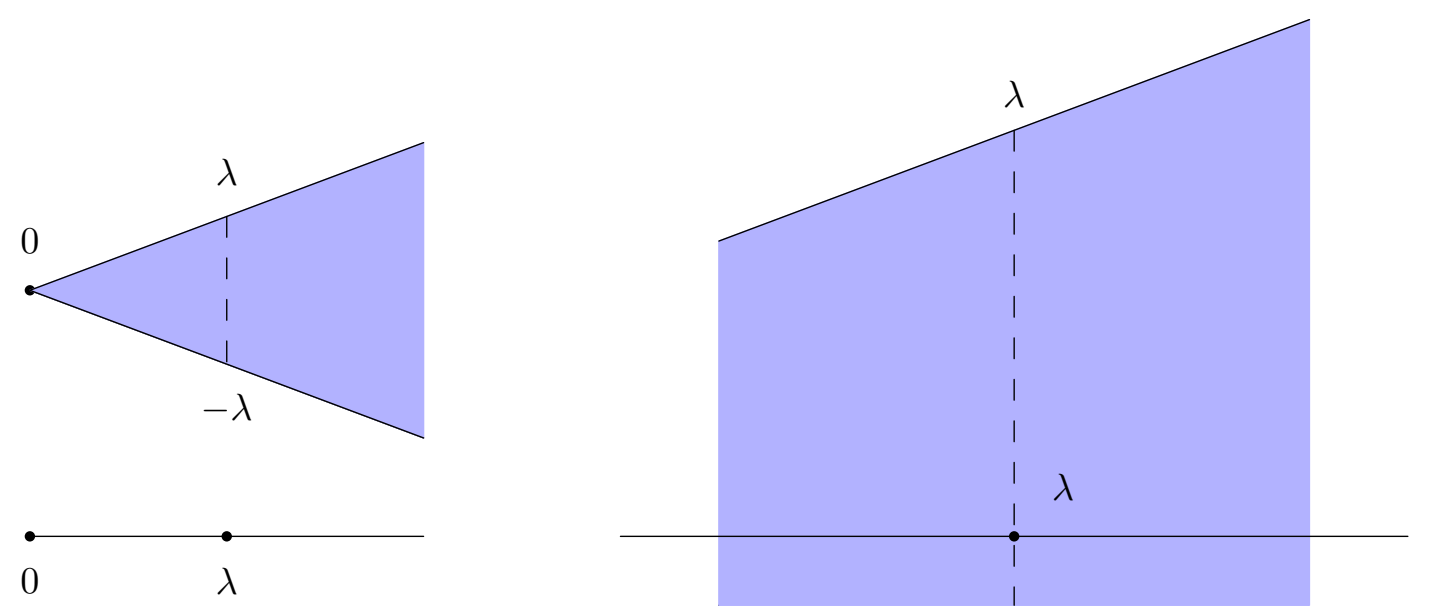

Figure 7.1. Universal Weyl polyhedra and their fibers over the $J$ dominant chambers. Here $\mathfrak{g}=\mathfrak{s l}_{2}$, and $J=I$, $\emptyset$, i.e. full and empty integrabilities.

\section{WEAK FAMILIES OF WEYL POLYHEDRA AND CONCAVITY}

We continue to fix an arbitrary Kac-Moody algebra $\mathfrak{g}$ and a subset $J \subset I$ of simple roots. Recall that in the definition of a family of Weyl polyhedra $\pi: \mathcal{P} \rightarrow S$, we asked that for $s_{0}, s_{1} \in S, 0 \leqslant t \leqslant 1$, we have:

$$
\pi^{-1}\left(t s_{0}+(1-t) s_{1}\right)=t \pi^{-1} s_{0}+(1-t) \pi^{-1} s_{1}
$$

In this section, we relax this condition, and relate a more flexible notion of a family of Weyl polyhedra to certain maps to the $J$ dominant chamber. Along the way, we prove new results even about the convex hulls of highest weight modules, cf. Proposition 8.14.

Definition 8.1. Let $S$ be a convex set. By a weak $S$-family of Weyl polyhedra we mean a subset $\mathcal{P} \subset S \times \mathfrak{h}^{*}$ such that (i) under the natural projection $\pi: \mathcal{P} \rightarrow S$, for each $s \in S$ the fiber $\pi^{-1} s$ is a Weyl polyhedron, and (ii) $\mathcal{P}$ is convex.

See Figure 8.1 for an example of an $S$-family and a weak $S$-family. As before, we have:

Lemma 8.2. If $S^{\prime} \rightarrow S$ is a morphism of convex sets, and $\mathcal{P} \rightarrow S$ a weak $S$-family of Weyl polyhedra, then the pullback $\mathcal{P} \times{ }_{S} S^{\prime}$ is a weak $S^{\prime}$-family of Weyl polyhedra.

The goal in this section is to relate weak families to certain maps to $D_{J}$, in the spirit of Theorem 6.4. To this end, we will need a more explicit description of the $J$ dominant points of $P(\lambda, J)$, in the spirit of Proposition 3.7 and [22, Proposition 
FACES OF HIGHEST WEIGHT MODULES AND THE UNIVERSAL WEYL POLYHEDRON 31
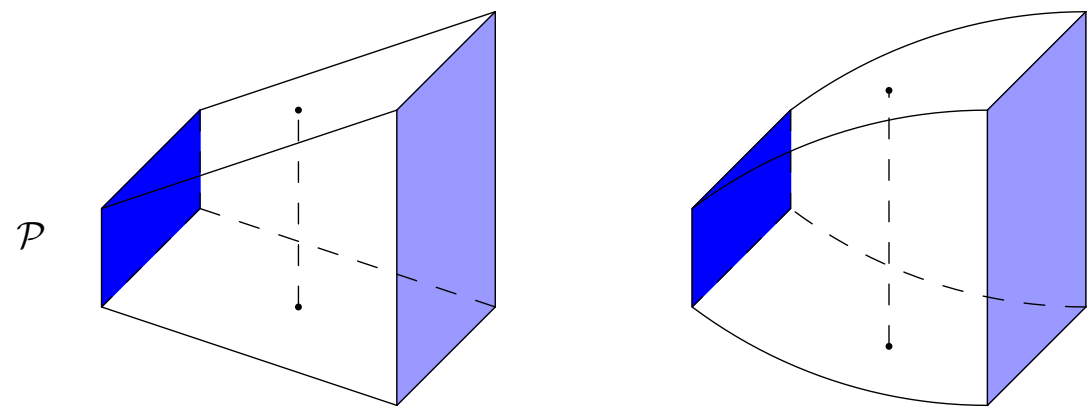

$S$
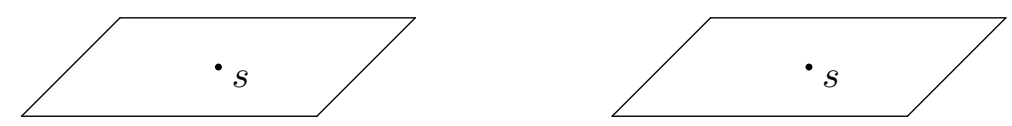

Figure 8.1. Examples of an $S$ family and a weak $S$ family of Weyl polyhedra, respectively, for $\mathfrak{g}=\mathfrak{s l}_{2}$ and $J=I$. The fiber in either case is the segment between $(s, \lambda(s))$ and $(s,-\lambda(s))$.

2.4]. After collecting some helpful ingredients, this description will be obtained in Theorem 8.16

Definition 8.3. Let $\lambda, \nu \in \mathfrak{h}^{*}$. We say $\nu$ is $J$ nondegenerate with respect to $\lambda$ if the following two conditions hold:

(1) $\lambda-\nu \in \mathbb{R}^{\geqslant 0} \pi$,

(2) write $\lambda-\nu=\beta_{J}+\beta_{I \backslash J}, \beta_{J} \in \mathbb{R}^{\geqslant 0} \pi_{J}, \beta_{I \backslash J} \in \mathbb{R}^{\geqslant 0} \pi_{I \backslash J}$. Then for any connected component $C$ of $\operatorname{supp} \beta_{J}$, there exists $c \in C$ such that $\left(\check{\alpha}_{c}, \lambda-\right.$ $\left.\beta_{I \backslash J}\right)>0$.

For brevity, we will write $\nu \leqslant_{J} \lambda$ if $\nu$ is $J$ nondegenerate with respect to $\lambda$.

Remark 8.4. One can show that $\leqslant_{J}$ is the usual partial order on $\mathfrak{h}^{*}$ if and only if $J$ is empty.

Remark 8.5. If $\lambda, \nu$ are $J$ dominant and $\lambda$ has finite stabilizer in $W_{J}$, then condition (2) is automatic.

To relate weak families to maps to $D_{J}$, we introduce the following class of maps.

Definition 8.6. Let $S$ be a convex set. We say a map $f: S \rightarrow D_{J}$ is concave if for all $s_{1}, s_{2} \in S, 0 \leqslant t \leqslant 1$, we have:

$$
t f\left(s_{1}\right)+(1-t) f\left(s_{2}\right) \leqslant_{J} f\left(t s_{1}+(1-t) s_{2}\right),
$$

i.e. the secant line lies below the graph in the partial order $\leqslant_{J}$. 
Notice that concave maps pull back under morphisms of convex sets:

Lemma 8.7. If $\phi: S^{\prime} \rightarrow S$ is a morphism of convex sets, and $f: S \rightarrow D_{J}$ a concave map. Then $f \circ \phi: S^{\prime} \rightarrow D_{J}$ is concave.

It follows that the assignment $S \rightsquigarrow\left\{\right.$ concave maps $\left.S \rightarrow D_{J}\right\}$ is a contravariant functor from convex sets to sets. We can now state the main result of this section.

Theorem 8.8. There is an isomorphism of functors:

$\{$ weak $S$-families of Weyl polyhedra $\} \simeq\left\{\right.$ concave maps $\left.S \rightarrow D_{J}\right\}$.

The remainder of this section is devoted to proving Theorem 8.8. Note that as the terminology suggests, we have:

Lemma 8.10. $\leqslant_{J}$ is a partial order on $\mathfrak{h}^{*}$.

Proof. We only explain transitivity, as the other properties are immediate. Suppose $\mu \leqslant J \nu \leqslant_{J} \lambda$. Write:

$$
\begin{array}{ll}
\lambda=\nu+\beta_{J}+\beta_{I \backslash J}, & \beta_{J} \in \mathbb{R}^{\geqslant 0} \pi_{J}, \quad \beta_{I \backslash J} \in \mathbb{R}^{\geqslant 0} \pi_{I \backslash J}, \\
\nu=\mu+\gamma_{J}+\gamma_{I \backslash J}, & \gamma_{J} \in \mathbb{R}^{\geqslant 0} \pi_{J}, \quad \gamma_{I \backslash J} \in \mathbb{R}^{\geqslant 0} \pi_{I \backslash J} .
\end{array}
$$

We need to show that for any connected component $C$ of $\operatorname{supp}\left(\beta_{J}+\gamma_{J}\right)$, there exists $c \in C$ with $\left(\check{\alpha}_{c}, \lambda-\beta_{I \backslash J}-\gamma_{I \backslash J}\right)>0$. If $C$ contains a connected component of $\operatorname{supp} \beta_{J}$, then we may pick $c \in C$ with $\left(\check{\alpha}_{c}, \lambda-\beta_{I \backslash J}\right)>0$, whence:

$$
\left(\check{\alpha}_{c}, \lambda-\beta_{I \backslash J}-\gamma_{I \backslash J}\right) \geqslant\left(\check{\alpha}_{c}, \lambda-\beta_{I \backslash J}\right)>0 .
$$

If $C$ contains no components of $\operatorname{supp} \beta_{J}$, then we have $\left(\check{\alpha}_{c}, \lambda-\beta_{I \backslash J}\right)=\left(\check{\alpha}_{c}, \nu\right)$, $\forall c \in C$. Thus if we pick $c \in C$ with $\left(\check{\alpha}_{c}, \nu-\gamma_{I \backslash J}\right)>0$, we have:

$$
\left(\check{\alpha}_{c}, \lambda-\beta_{I \backslash J}-\gamma_{I \backslash J}\right)=\left(\check{\alpha}_{c}, \nu-\gamma_{I \backslash J}\right)>0 .
$$

We also note the following:

Lemma 8.11. For $\lambda \in D_{J}$, the locus $\left\{\nu \in \mathfrak{h}^{*}: \nu \leqslant J\right\}$ is convex and forms a cone with vertex at $\lambda$.

We now obtain the desired description of $P(\lambda, J)$ in the case $J=I$.

Proposition 8.12. For a point $\lambda$ in the dominant chamber $D_{I}$, we have:

$$
\operatorname{conv} W \lambda=\bigcup_{w \in W} w\left\{\nu \in D_{I}: \nu \leqslant I \lambda\right\} .
$$

Proof. For the inclusion $\subset$, it suffices to show that $\nu \leqslant_{I} \lambda$ for any point $\nu$ of conv $W \lambda$. We first show $w \lambda \leqslant_{I} \lambda$ by induction on $\ell(w)$. The case $w=1$ is trivial, and for the inductive step write $w=s_{i} w^{\prime}$, where $\ell\left(w^{\prime}\right)=\ell(w)-1$. As $\lambda$ is dominant, we have $w \lambda \leqslant w^{\prime} \lambda$, and by considering $\check{\alpha}_{i}$ we have $w \lambda \leqslant \leqslant_{I} w^{\prime} \lambda$. We therefore are done 
by induction and Lemma 8.10. Having shown $w \lambda \leqslant_{I} \lambda, \forall w \in W$, we are done by Lemma 8.11,

For the inclusion $\supset$, it suffices to show that for $\nu \in D_{I}, \nu \leqslant_{I} \lambda$, we have $\nu \in$ conv $W \lambda$. We will in fact show:

For $\mu \leqslant_{I} \eta, J:=\operatorname{supp}(\eta-\mu)$, if $\mu$ and $\eta$ are $J$ dominant then $\mu \in \operatorname{conv} W_{J}(\eta)$.

Let $C$ be a connected component of $J$, and write $\eta-\mu=\beta_{C}+\beta_{J \backslash C}$, where $\beta_{C} \in \mathbb{R}^{>0} \pi_{C}, \beta_{J \backslash C} \in \mathbb{R}^{>0} \pi_{J \backslash C}$. If we can show that $\eta-\beta_{C}$ lies in conv $W_{C}(\eta)$, then $\eta-\beta_{C}, \mu$ again satisfy the conditions of Equation (8.13), so we are done by induction on the cardinality of $J$.

So, it remains to show Equation (8.13) when $J$ is connected. We break into two cases. If there exists $j \in J$ with $\left(\check{\alpha}_{j}, \mu\right)>0$, then the desired claim is shown in the second and third paragraph of [22, Proposition 2.4].

If $\left(\check{\alpha}_{j}, \mu\right)=0, \forall j \in J$, we argue as follows. Since $\mu \leqslant I \eta$, for some $j_{0} \in J$ we have $\left(\check{\alpha}_{j}, \eta\right)>0$. Thus $\eta-\mu$ is a positive real combination of simple roots satisfying $\left(\check{\alpha}_{j}, \eta-\mu\right) \geqslant 0, \forall j \in J,\left(\check{\alpha}_{j_{0}}, \eta-\mu\right)>0$, whence $J$ is of finite type. It therefore remains to show that if $\mathfrak{g}$ is a simple Lie algebra, $\lambda$ a real dominant weight, then $0 \in \operatorname{conv} W(\lambda)$. But this follows from $W$-invariance:

$$
0=\frac{1}{|W|} \sum_{w \in W} w \lambda
$$

To obtain the desired description of $P(\lambda, J)$ for general $J$, we need one more preliminary, which is the convex analogue of the Integrable Slice Decomposition 3.14 .

Proposition 8.14. For any $J \subset I$, and $\lambda \in D_{J}$, we have:

$$
P(\lambda, J)=\bigsqcup_{\mu \in \mathbb{R} \geqslant 0} \operatorname{conv} W_{J}(\lambda-\mu) .
$$

Proof. The inclusion $\supset$ is straightforward from the definition of $P(\lambda, J)$. For the inclusion $\subset$, write an element of the left hand side as:

$$
\nu=\sum_{m} t_{m} w_{m}\left(\lambda-r_{m} \alpha_{i_{m}}\right), \quad t_{m} \geqslant 0, \sum_{m} t_{m}=1, r_{m} \geqslant 0, i_{m} \in I \backslash J .
$$

By $W_{J}$ invariance, we may assume $\nu$ is $J$ dominant, whence by Proposition 8.12 applied to $\mathfrak{g}_{J}$ it suffices to show $\nu$ is $J$ nondegenerate with respect to $\lambda-$ $\sum_{m} t_{m} r_{m} \alpha_{i_{m}}$. This in turn follows by using that for each $m, w_{m}\left(\lambda-r_{m} \alpha_{i_{m}}\right)$ is $J$ nondegenerate with respect to $\lambda-r_{m} \alpha_{i_{m}}$, as was shown in the proof of Proposition 8.12 .

Finally, we obtain the desired alternative description of $P(\lambda, J)$ in terms of $\leqslant J$ : 
Theorem 8.16. For any $J \subset I, \lambda \in D_{J}$, we have:

$$
P(\lambda, J)=\bigcup_{w \in W_{J}} w\left\{\nu \in D_{J}: \nu \leqslant J \lambda\right\}
$$

Proof. This is immediate from Propositions 8.12 and 8.14

Proof of Theorem 8.8. The maps between the two sides will be exactly as in Theorem 6.4. We need only to see that the property that the total space of a family $\mathcal{P}$ be convex is equivalent to concavity of the corresponding map $\phi_{\mathcal{P}}$ to $D_{J}$. Let $s_{0}, s_{1}$ be two distinct points of $S$, and $P\left(\lambda_{0}, J\right), P\left(\lambda_{1}, J\right)$ the corresponding fibers. A direct calculation shows:

$\operatorname{conv}\left(s_{0} \times P\left(\lambda_{0}, J\right) \sqcup s_{1} \times P\left(\lambda_{1}, J\right)\right)=\bigsqcup_{0 \leqslant t \leqslant 1}\left(t s_{0}+(1-t) s_{1}\right) \times P\left(t \lambda_{0}+(1-t) \lambda_{1}, J\right)$.

Therefore the condition that the total space is convex is that $P\left(t \lambda_{0}+(1-t) \lambda_{1}\right) \subset$ $P\left(\lambda\left(t s_{0}+(1-t) s_{1}\right), J\right)$. That this is equivalent to the concavity of $\phi_{\mathcal{P}}$ follows from the following Lemma 8.18 .

Lemma 8.18. Let $\mu, \nu \in D_{J}$. Then $P(\mu, J) \subset P(\nu, J)$ if and only if $\mu \leqslant_{J} \nu$.

We note that for $\mathfrak{g}$ of finite type, and $\mu, \nu$ dominant integral weights, Lemma 8.18 can be found in [16, Proposition 2.2].

Proof. If $P(\mu, J) \subset P(\nu, J)$, in particular $\mu \in P(\nu, J)$, whence we are done by Theorem 8.16. Conversely, suppose $\mu \leqslant{ }_{J} \nu$. By $W_{J}$ invariance it suffices to show that every $J$ dominant weight $\eta$ of $P(\mu, J)$ lies in $P(\nu, J)$. To see this, again by Theorem $8.16 \eta \leqslant_{J} \mu$, whence $\eta \leqslant_{J} \nu$ by Lemma 8.10, and so $\eta \in P(\nu, J)$ by Theorem 8.16 .

Remark 8.19. Using results from related work [8], specifically, Propositions 3.7 and 3.14, one similarly obtains a description of the weights of parabolic Verma modules $M(\lambda, J)$. Let us write $\leqslant_{J}^{\prime}$ for the variant on $\leqslant_{J}$ given by replacing $\mathbb{R}^{\geqslant 0} \pi$ by $\mathbb{Z}^{\geqslant 0} \pi$ in Definition 8.3, Then:

$$
\text { wt } M(\lambda, J)=\bigcup_{w \in W_{J}} w\left\{\nu \in P_{J}^{+}: \nu \leqslant_{J}^{\prime} \lambda\right\}
$$

By another result of loc. cit., for $L(\lambda)$ a simple highest weight module, and $I_{L(\lambda)}:=$ $\left\{i \in I:\left(\check{\alpha}_{i}, \lambda\right) \in \mathbb{Z}^{\geqslant 0}\right\}$, we have:

$$
\operatorname{wt} L(\lambda)=\bigcup_{w \in W_{I_{L}(\lambda)}} w\left\{\nu \in P_{I_{L(\lambda)}}^{+}: \nu \leqslant_{I_{L(\lambda)}}^{\prime} \lambda\right\} .
$$




\section{Closure, polyhedrality, ANd integrable isotropy}

In this section we turn to the question of polyhedrality of conv $V$. As we have seen, conv $V=$ conv $M\left(\lambda, I_{V}\right)$, so it suffices to consider parabolic Verma modules. If the Dynkin diagram $I$ of $\mathfrak{g}$ can be factored as a disconnected union $I=I^{\prime} \sqcup I^{\prime \prime}$, in the obvious notation we have corresponding factorizations:

$$
M\left(\lambda^{\prime} \oplus \lambda^{\prime \prime}, J^{\prime} \sqcup J^{\prime \prime}\right) \simeq M\left(\lambda^{\prime}, J^{\prime}\right) \otimes M\left(\lambda^{\prime \prime}, J^{\prime \prime}\right),
$$

so it suffices to consider $\mathfrak{g}$ indecomposable. As is clear from the Ray Decomposition 3.17, in finite type conv $V$ is always a polyhedron, so throughout this section we take $\mathfrak{g}$ to be indecomposable of infinite type.

We first show that conv $V$ is almost never a polytope. Let us call $V$ trivial if $[\mathfrak{g}, \mathfrak{g}]$ acts by 0 , i.e. $V$ is one-dimensional.

Proposition 9.1. conv $V$ is a polytope if and only if $V$ is trivial.

Proof. One direction is tautological. For the reverse, if conv $V$ is a polytope, then $V$ is integrable. Assume first that $V$ is simple; then $V$ is the inflation of a $\overline{\mathfrak{g}}$-module, where $\overline{\mathfrak{g}}$ is as in Section 3.1. Now recall that if $\mathfrak{i}$ is an ideal of $\overline{\mathfrak{g}}$, then either $\mathfrak{i}$ is contained in the (finite-dimensional) center of $\overline{\mathfrak{g}}$ or contains $[\overline{\mathfrak{g}}, \overline{\mathfrak{g}}]$. Since the kernel of $\overline{\mathfrak{g}} \rightarrow \operatorname{End}(V)$ has finite codimension, the claim follows. For the case of general integrable $V$, we still know $V$ has a unique weight by Proposition 3.7. Now the result follows by recalling that $[\mathfrak{g}, \mathfrak{g}] \cap \mathfrak{h}$ is spanned by $\check{\alpha}_{i}, i \in I$.

Even if we relax the assumption of boundedness, it transpires that $\operatorname{conv} V$ is only a polyhedron in fairly degenerate circumstances:

Proposition 9.2. Let $V$ be a non-trivial module. Then conv $V$ is a polyhedron if and only if $I_{V}$ corresponds to a Dynkin diagram of finite type.

Proof. If $I_{V}$ corresponds to a Dynkin diagram of finite type, we are done by the Ray Decomposition 3.17. Now suppose $I_{V}$ is not a diagram of finite type. If the orbit $W_{I_{V}} \lambda$ is infinite, we deduce that conv $V$ has infinitely many 0 -faces, whence it is not a polyhedron. If instead the orbit is finite, we deduce that the $\mathfrak{l}_{I_{V}}$ module of highest weight $\lambda$ is finite-dimensional, whence by the same reasoning as Proposition 9.1, the orbit is a singleton. In this case, by the non-triviality of $V$ and indecomposability of $\mathfrak{g}$ we may choose $i \in I \backslash I_{V}$ which is connected to a component of $I_{V}$ of infinite type. It follows by the previous case that the orbit of $\lambda-\alpha_{i}$ is infinite. Hence by the Ray Decomposition 3.17, conv $V$ has infinitely many 1-faces, whence it is not a polyhedron.

In particular, we have shown:

Corollary 9.3. For any $V$ in finite type, conv $V$ is the Minkowski sum of conv $W_{I_{V}}(\lambda)$ and the cone $\mathbb{R}^{\geqslant 0} W_{I_{V}}\left(\pi_{I \backslash I_{V}}\right)$. 
This corollary extends the notion of the Weyl polytope of a finite-dimensional simple representation, to the Weyl polyhedron of any highest weight module $V$ in finite type.

Recall that a set $X \subset \mathfrak{h}^{*}$ is locally polyhedral if its intersection with every polytope is a polytope. It turns out that for generic $V, \operatorname{conv} V$ is locally polyhedral, as we show in the main theorem of this section.

Theorem 9.4. Let $V$ be a non-trivial module. The following are equivalent:

(1) conv $V$ is locally polyhedral.

(2) The tangent cone $T_{\lambda} V$ is closed, and conv $V=\bigcap_{w \in W_{I_{V}}} T_{w \lambda} V$.

(3) conv $V$ is closed.

(4) The stabilizer of $\lambda$ in $W_{I_{V}}$ is finite.

(5) $\lambda$ lies in the interior of the $I_{V}$ Tits cone.

Remark 9.5. The equivalence of (4) and (5) was already recalled in Proposition 3.2. and we include (5) only to emphasize the genericity of these conditions. In the integrable case, i.e. $I_{V}=I$, Borcherds in [2] considers the very same subset of the dominant chamber, which he calls $C_{\Pi}$, and writes:

The Tits cone can be thought of as obtained from $W_{\Pi} C_{\Pi}$ by "adding some boundary components".

Proof of Theorem 9.4. We may assume $V$ is a parabolic Verma module. As remarked above, (4) and (5) are equivalent. We will show the following sequence of implications:

$$
(1) \Longrightarrow(3) \Longrightarrow(4) \Longrightarrow(2) \Longrightarrow(3), \text { and }(2)-(5) \Longrightarrow(1)
$$

It is easy to see (1) implies (3) by intersecting with cubes of side-length tending to infinity. We now show the equivalence of (2), (3), and (4). The implication (2) implies (3) is tautological. To show that (3) implies (4), write $J=\left\{j \in I_{V}\right.$ : $\left.\left(\check{\alpha}_{j}, \lambda\right)=0\right\}$ as in Lemma 4.4 and recall by Proposition 3.2 that the stabilizer of $\lambda$ is $W_{J}$. Suppose that $W_{J}$ is infinite, and choose a connected sub-diagram $J^{\prime} \subset J$ of infinite type. By the non-triviality of $V$, and the indecomposability of $\mathfrak{g}$, we may choose $i \in I \backslash J$ which shares an edge with $J^{\prime}$. It follows that $\lambda-\alpha_{i}$ is a weight of $V$, and $U\left(\mathfrak{l}_{J^{\prime}}\right) V_{\lambda-\alpha_{i}}$ is a non-trivial integrable highest weight $\mathfrak{l}_{J^{\prime}}$-module. We may choose an imaginary positive root $\delta$ of $\mathfrak{l}_{J^{\prime}}$ with $\operatorname{supp} \delta=J^{\prime}$, cf. [13, Theorem 5.6 and $\S 5.12$. By Proposition [3.7, $\lambda-\alpha_{i}-\mathbb{Z}^{\geqslant 0} \delta$ lies in wt $V$. It follows that $\lambda-\mathbb{Z}^{\geqslant 0} \delta$ lies in the closure of conv $V$, but cannot lie in conv $V$ by Equation (3.16). It may be clarifying for the reader to visualize this argument using Figure 3.1.

We next show that (4) implies (2). By Lemma 4.4 and the Ray Decomposition 3.17, our assumption on $\lambda$ implies the tangent cone $T_{\lambda} V$ is a polyhedral cone, whence 
closed. It therefore remains to show:

$$
\operatorname{conv} V=\bigcap_{w \in W_{I_{V}}} T_{w \lambda} V
$$

The inclusion $\subset$ is tautological. For the reverse, writing $D$ for the $I_{V}$ dominant chamber, we first show $T_{\lambda} V \cap D \subset$ conv $V$, i.e. the left-hand side coincides with the intersection of the right-hand side with the $I_{V}$ Tits cone. Since $\left(\check{\alpha}_{j}, \lambda\right) \in \mathbb{Z}, \forall j \in I_{V}$, it is straightforward to see that the translate $\left(T_{\lambda} V \cap D\right)-\lambda$ is a rational polyhedron (cf. Proposition 3.4). In particular, it suffices to consider $v \in T_{\lambda} V \cap D$ of the form $v \in \lambda-\mathbb{Q}^{\geqslant 0} \pi$. As both sides of Equation (9.6) are dilated by $n$, when we pass from $M\left(\lambda, I_{V}\right)$ to $M\left(n \lambda, I_{V}\right)$, for any $n \in \mathbb{Z}^{>0}$, it suffices to consider $v \in T_{\lambda} V \cap D$ of the form $v \in \lambda-\mathbb{Z}^{\geqslant 0} \pi$. Writing $v=\lambda-\mu^{\prime}-\mu^{\prime \prime}$, where $\mu^{\prime} \in \mathbb{Z}^{\geqslant 0} \pi_{I_{V}}, \mu^{\prime \prime} \in \mathbb{Z}^{\geqslant 0} \pi_{I \backslash I_{V}}$, it suffices to see that $v$ is non-degenerate with respect to $\lambda-\mu^{\prime \prime}$. But this follows since the stabilizer of $\lambda-\mu^{\prime \prime}$ is again of finite type by Proposition 3.7(2).

It remains to show the right-hand side lies in the $I_{V}$ Tits cone. Looijenga has shown in [22, Corollary 2.5] that if $K \subset D$ is a compact subset of the interior of the $I_{V}$ Tits cone, then conv $W_{I_{V}}(K)$ is closed. To apply this in our situation, introduce the following 'cutoffs' of the Ray Decomposition 3.17.

$$
K_{t}:=\bigcup_{i \in I \backslash I_{V}} \lambda-[0, t] \alpha_{i}, \quad t \geqslant 0 .
$$

By filtering $\mathfrak{h}^{*}$ by half spaces of the form $\{(\mu,-) \geqslant t\}$, where $\mu=\sum_{i \in I \backslash I_{V}} \check{\omega}_{i}$, it suffices to know that conv $W_{I_{V}}\left(K_{t}\right)$ is closed for all $t \geqslant 0$; but this follows from Looijenga's result.

Continuing with the proof, if the right-hand side of Equation (9.6) contained a point $x$ outside of the $I_{V}$ Tits cone, consider the line segment $\ell=[\lambda, x]$. The intersection of $\ell$ with the $I_{V}$ Tits cone is closed and convex, whence of the form $[\lambda, y]$. But now $y$ is a point of $\operatorname{conv} V$ which lies on the boundary of the $I_{V}$ Tits cone. In particular, conv $V$ is not contained in the interior of the $I_{V}$ Tits cone, which contradicts (5).

It remains to see the equivalent conditions (2)-(5) imply (1). For this we will introduce a convexity-theoretic analogue of the principal gradation, cf. [13, §10.8]. Choose $\check{\rho}$ in the real dual of $\mathfrak{h}^{*}$ satisfying $\left(\check{\rho}, \alpha_{i}\right)=1, \forall i \in I$, and for $A \subset \mathbb{R}$ write $H_{A}:=\check{\rho}^{-1}(A)$. In particular, write $H_{[k, \infty)}$ for the half-space $\check{\rho}^{-1}([k, \infty))$, and $V_{[k, \infty)}:=(\operatorname{conv} V) \cap H_{[k, \infty)}$. It suffices to check that $V_{[k, \infty)}$ is a polytope, for all $k \in \mathbb{R}$. Fix $k \in \mathbb{R}$, which we may assume to satisfy $k \leqslant \check{\rho}(\lambda)$, and note wt $V \cap H_{[k, \infty)}$ consists of finitely many points. Let $v_{i}, 1 \leqslant i \leqslant n$ be an enumeration of $W_{I_{V}}(\lambda) \cap H_{(k, \infty)}$. For each $v_{i}$, by (4) and the Ray Decomposition 3.17, let $f_{i j}$ denote the finitely many 1 -faces containing $v_{i}$. For example, by Theorem 4.2 if 
$v_{i}=\lambda$, these 1 -faces are

$$
w\left\{\lambda-\mathbb{R}^{\geqslant 0} \alpha_{i}\right\} \text { and } w\left[\lambda, s_{j}(\lambda)\right], \quad \text { where } \quad w \in \operatorname{Stab}_{W_{I_{V}}}(\lambda), i \in I \backslash I_{V}, j \in I_{V} .
$$

Here $[a, b]$ denotes the closed line segment joining $a$ and $b$. Each $f_{i j}$ intersects $H_{[k, \infty)}$ in a line segment, we define $e_{i j}$ to be the other endpoint, i.e. $\left[v_{i}, e_{i j}\right]:=f_{i j} \cap H_{[k, \infty)}$. By removing some $e_{i j}$, we may assume $e_{i j} \neq v_{k}, \forall i, j, k$. It suffices to show:

$$
\operatorname{conv} V_{[k, \infty)}=\operatorname{conv}\left\{v_{i}, e_{i j}\right\} \text {. }
$$

Writing $H_{k}$ for the affine hyperplane $\check{\rho}^{-1}(k)$, we claim that $H_{k} \cap \operatorname{conv} V=\operatorname{conv} e_{i j}$. We first observe that the intersection is a bounded subset of $H_{k}$. Indeed, it suffices to check $H_{k} \cap T_{\lambda} V$ is bounded, but $T_{\lambda} V$ by Lemma 4.4 is of the form $\operatorname{conv}\{\lambda-$ $\left.\mathbb{R}^{\geqslant 0} r_{k}\right\}_{1 \leqslant k \leqslant m}$, with $\check{\rho}\left(r_{k}\right)>0,1 \leqslant k \leqslant m$. So $H_{k} \cap \operatorname{conv} V$ is bounded, and closed by (2), whence it is the convex hull of its 0-faces, cf. [10, §2.4.5]. But if $v$ is an interior point of a $d$-face of conv $V, d \geqslant 2$, then $v$ cannot be a 0 -face of a hyperplane section. Hence the 0 -faces of $H_{k} \cap \operatorname{conv} V$ are found by intersecting the 0 -faces and 1-faces of conv $V$ with $H_{k}$, which is exactly the collection $e_{i j}$.

We are ready to show that $V_{[k, \infty)}=\operatorname{conv}\left\{v_{i}, e_{i j}\right\}$. The inclusion $\supset$ is immediate. For the inclusion $\subset$, write $v \in V_{[k, \infty)}$ as a convex combination:

$$
v=\sum_{r^{\prime} \in R^{\prime}} t_{r^{\prime}} r^{\prime}+\sum_{r^{\prime \prime} \in R^{\prime \prime}} t_{r^{\prime \prime}} r^{\prime \prime}
$$

where $r^{\prime}, r^{\prime \prime}$ are points along the rays occurring in the Ray Decomposition 3.17 satisfying $\check{\rho}\left(r^{\prime}\right) \geqslant k>\check{\rho}\left(r^{\prime \prime}\right)$, respectively. For $r^{\prime} \in R^{\prime}$, by construction we can write $r^{\prime}$ as a convex combination of $v_{i}, e_{i j}$. Observe $R^{\prime}$ must be non-empty, else $\check{\rho}(v)<k$. If $R^{\prime \prime}$ is also non-empty, define the partial averages:

$$
v^{\prime}:=\frac{1}{\sum_{s^{\prime} \in R^{\prime}} t_{s^{\prime}}} \sum_{r^{\prime} \in R^{\prime}} t_{r^{\prime}} r^{\prime}, \quad v^{\prime \prime}:=\frac{1}{\sum_{s^{\prime \prime} \in R^{\prime \prime}} t_{s^{\prime \prime}}} \sum_{s^{\prime \prime} \in R^{\prime \prime}} t_{r^{\prime \prime}} r^{\prime \prime} .
$$

The line segment $\left[v^{\prime}, v^{\prime \prime}\right]$ is not a single point, as $\check{\rho}\left(v^{\prime \prime}\right)<k$. Writing $v^{\prime \prime \prime}$ for its intersection with $H_{k}$, we have $v$ lies in $\left[v^{\prime}, v^{\prime \prime \prime}\right]$. But we already saw $v^{\prime} \operatorname{lies} \operatorname{in} \operatorname{conv}\left\{v_{i}, e_{i j}\right\}$, and $v^{\prime \prime \prime} \in \operatorname{conv}\left\{e_{i j}\right\}$ by the preceding paragraph; hence, $v \in \operatorname{conv}\left\{v_{i}, e_{i j}\right\}$ as desired.

Remark 9.7. It would be interesting to know whether the intersection formula in Theorem 9.4(2) holds without finite stabilizers.

As an application of Theorem 9.4, we provide a half-space representation of conv $V$ for generic highest weights, valid in any type.

Proposition 9.8. When it is closed, conv $V$ coincides with the intersection of the half-spaces

$$
H_{i, w}:=\left\{\mu \in \lambda-\mathbb{R} \pi:\left(w \lambda-\mu, w \check{\omega}_{i}\right) \geqslant 0\right\}, \quad \forall w \in W_{I_{V}}, i \in I .
$$


The above half-space representation extends results in finite type proved in [7, 18]; moreover, our proof uses tangent cones and is thus different from loc. cit.

Proof. One inclusion is clear, since wt $V$ is contained in each half-space $H_{i, w}$, by $W_{I_{V}}$-invariance. Conversely, by Theorem 9.4 we need to show that the intersection

$$
\bigcap_{i \in I} \bigcap_{w \in W_{I_{V}}} H_{i, w}
$$

is contained in the tangent cone $T_{\lambda} V$. Since $\lambda$ has finite stabilizer in $W_{I_{V}}$, the cone $T_{\lambda} V$ is a convex polyhedron (e.g. by Lemma 4.4). Hence it is the intersection of the half-spaces that define its facets. Now observe that each of these half-spaces appears in the above intersection.

\section{A question of Brion on localization of CONVEX Hulls}

We now return to the case of an arbitrary Kac-Moody Lie algebra $\mathfrak{g}$ and answer the question of Brion [4] mentioned in Section 2.

Theorem 10.1. Suppose $\mathfrak{g}$ is arbitrary. Fix $\lambda, V$ with $\left(\check{\alpha}_{i}, \lambda\right)>0$ for all $i \in I_{V}$. Given a standard face $F=\mathrm{wt}_{l_{J}}$ for some $J \subset I$, and setting $J^{\prime}:=I_{V} \cap J$, we have

$$
\operatorname{Loc}_{J} \operatorname{conv} V=\bigcap_{w \in W_{J^{\prime}}} T_{w \lambda} V=\operatorname{conv} M\left(\lambda, J^{\prime}\right) .
$$

Note that the localization of conv $V$ at other faces $F$ is computed by Theorem 4.2 .

Proof. Given distinct points $a, b$ in any convex set $C$ in a real vector space, if the line segment $[a, b]$ can be slightly extended in $C$ past $b$, namely $a+(1+\epsilon)(b-a) \in C$ for some $\epsilon>0$, then $T_{a} C \subset T_{b} C$. This assertion can be checked by a computation in the plane.

By the above observation and the Ray Decomposition 3.17, $\operatorname{Loc}_{J}$ conv $V$ equals the intersection over the tangent cones at the vertices in the face, i.e. $W_{J^{\prime}}(\lambda)$. Since $\lambda$ is $I_{V}$-regular, these coincide with the tangent cones for $M\left(\lambda, J^{\prime}\right)$ at these points, cf. Lemma 4.4. We are then done by Theorem 9.4(2).

Remark 10.3. While the results in this paper are developed for $\mathfrak{g}$-modules, they also hold for $\overline{\mathfrak{g}}$-modules, where $\overline{\mathfrak{g}}$ is as in Section 3.1. That the results cited from the literature and our previous work [8] apply for $\overline{\mathfrak{g}}$ was explained in loc. cit. With this, the arguments in the present paper apply verbatim for $\overline{\mathfrak{g}}$, except for Propositions 9.1 and 9.2 , which were shown for both $\mathfrak{g}$ and $\overline{\mathfrak{g}}$. 


\section{Highest Weight MODUlES OVER SYMMETRIZABLE QUANTUM GROUPS}

The goal of this section is to prove Theorems 5.1 and 5.7 for modules over quantum groups $U_{q}(\mathfrak{g})$, for $\mathfrak{g}$ a Kac-Moody algebra. Given a generalized Cartan matrix $A$, as for $\mathfrak{g}=\mathfrak{g}(A)$, to write down a presentation for the algebra $U_{q}(\mathfrak{g})$ via generators and explicit relations, one uses the symmetrizability of $A$. When $\mathfrak{g}$ is non-symmetrizable, even the formulation of $U_{q}(\mathfrak{g})$ is subtle and is the subject of active research [15, 9]. In light of this, we restrict to $U_{q}(\mathfrak{g})$ where $\mathfrak{g}$ is symmetrizable.

11.1. Notation and preliminaries. We begin by reminding standard definitions and notation. Fix $\mathfrak{g}=\mathfrak{g}(A)$ for $A$ a symmetrizable generalized Cartan matrix. Fix a diagonal matrix $D=\operatorname{diag}\left(d_{i}\right)_{i \in I}$ such that $D A$ is symmetric and $d_{i} \in \mathbb{Z}^{>0}, \forall i \in I$. Let $(\mathfrak{h}, \pi, \check{\pi})$ be a realization of $A$ as before; further fix a lattice $P^{\vee} \subset \mathfrak{h}$, with $\mathbb{Z}$-basis $\check{\alpha}_{i}, \check{\beta}_{l}, i \in I, 1 \leqslant l \leqslant|I|-\operatorname{rk}(A)$, such that $P^{\vee} \otimes_{\mathbb{Z}} \mathbb{C} \simeq \mathfrak{h}$ and $\left(\check{\beta}_{l}, \alpha_{i}\right) \in \mathbb{Z}, \forall i \in$ $I, 1 \leqslant l \leqslant|I|-\operatorname{rk}(A)$. Set $P:=\left\{\lambda \in \mathfrak{h}^{*}:\left(P^{\vee}, \lambda\right) \subset \mathbb{Z}\right\}$ to be the weight lattice. We further retain the notations $\rho, \mathfrak{b}, \mathfrak{h}, \mathfrak{l}_{J}, P_{J}^{+}, M(\lambda, J), I_{L(\lambda)}$ from previous sections; note we may and do choose $\rho \in P$. We normalize the Killing form $(\cdot, \cdot)$ on $\mathfrak{h}^{*}$ to satisfy: $\left(\alpha_{i}, \alpha_{j}\right)=d_{i} a_{i j}$ for all $i, j \in I$.

Let $q$ be an indeterminate. Then the corresponding quantum Kac-Moody algebra $U_{q}(\mathfrak{g})$ is a $\mathbb{C}(q)$-algebra, generated by elements $f_{i}, q^{h}, e_{i}, i \in I, h \in P^{\vee}$, with relations given in e.g. 11, Definition 3.1.1]. Among these generators are distinguished elements $K_{i}=q^{d_{i} \check{\alpha}_{i}} \in q^{P^{\vee}}$. Also define $U_{q}^{ \pm}$to be the subalgebras generated by the $e_{i}$ and the $f_{i}$, respectively.

A weight of the quantum torus $\mathbb{T}_{q}:=\mathbb{C}(q)\left[q^{P^{\vee}}\right]$ is a $\mathbb{C}(q)$-algebra homomorphism $\chi: \mathbb{T}_{q} \rightarrow \mathbb{C}(q)$, which we identify with an element $\mu_{q} \in\left(\mathbb{C}(q)^{\times}\right)^{2|I|-\operatorname{rk}(A)}$ given an enumeration of $\check{\alpha}_{i}, i \in I$. We will abuse notation and write $\mu_{q}\left(q^{h}\right)$ for $\chi_{\mu_{q}}\left(q^{h}\right)$. There is a partial ordering on the set of weights, given by: $q^{-\nu} \mu_{q} \leqslant \mu_{q}$, for all weights $\nu \in \mathbb{Z}^{\geqslant 0} \pi$.

Given a $U_{q}(\mathfrak{g})$-module $V$ and a weight $\mu_{q}$, the corresponding weight space of $V$ is:

$$
V_{\mu_{q}}:=\left\{v \in V: q^{h} v=\mu_{q}\left(q^{h}\right) v \forall h \in P^{\vee}\right\} .
$$

Denote by wt $V$ the set of weights $\left\{\mu_{q}: V_{\mu_{q}} \neq 0\right\}$. We say a $U_{q}(\mathfrak{g})$-module is highest weight if there exists a nonzero weight vector which generates $V$ and is killed by $e_{i}, \forall i \in I$. If we write $\lambda_{q}$ for the highest weight, the integrability of $V$ equals:

$$
I_{V}:=\left\{i \in I: \operatorname{dim} \mathbb{C}(q)\left[f_{i}\right] V_{\lambda_{q}}<\infty\right\} .
$$

11.2. Inclusions between faces of highest weight modules. For highest weight modules over quantum groups, notice convex hulls only make their appearance after specializing at $q=1$ [24]. Nonetheless, the above results give information on the 
action of quantized Levi subalgebras:

$$
\operatorname{wt}_{J} V:=\operatorname{wt} U_{q}\left(\mathfrak{l}_{J}\right) V_{\lambda_{q}}=(\text { wt } V) \cap q^{-\mathbb{Z}^{\geqslant 0} \pi_{J}} \lambda_{q},
$$

where $U_{q}\left(\mathfrak{l}_{J}\right)$ is the subalgebra of $U_{q}(\mathfrak{g})$ generated by $q^{P^{\vee}}$ and $e_{j}, f_{j}, j \in J$.

To prove the analogues of Theorems 5.1 and 5.7 over quantum groups, we first define the sets $J_{\min }, J_{\max }$ in the quantum setting. For $J \subset I$, call a node $j \in J$ active if either (i) $j \notin I_{V}$, or (ii) $j \in I_{V}$ and $\lambda_{q}\left(q^{\check{\alpha}_{j}}\right) \neq \pm 1$. Now decompose the Dynkin diagram corresponding to $J$ as a disjoint union of connected components, and let $J_{\min }$ denote the union of all components containing an active node. Finally, define:

$$
\begin{aligned}
J_{d} & :=\left\{j \in I_{V}: \lambda_{q}\left(q^{\check{\alpha}_{j}}\right)= \pm 1 \text { and } j \text { is not connected to } J_{\min }\right\}, \\
J_{\max } & :=J_{\min } \sqcup J_{d} .
\end{aligned}
$$

Theorem 11.3. Let $\lambda_{q}$ be arbitrary. Fix subsets $J, J^{\prime} \subset I$.

(1) $\mathrm{wt}_{J} V \subset \mathrm{wt}_{J^{\prime}} V$ if and only if $J_{\min } \subset J^{\prime}$.

(2) $\operatorname{wt}_{J} V=\mathrm{wt}_{J^{\prime}} V$ if and only if $J_{\min } \subset J^{\prime} \subset J_{\max }$.

The arguments of Theorems 5.1 and 5.7 apply verbatim, where one uses the representation theory of highest weight $U_{q}\left(\mathfrak{s l}_{2}\right)$-modules instead of that of $U\left(\mathfrak{s l}_{2}\right)$, cf. [12].

In particular, the two applications to branching, Corollaries 5.9 and 5.10, also have quantum counterparts.

\section{REFERENCES}

[1] Alexander Barvinok. Integer points in polyhedra. Zurich Lectures in Advanced Mathematics, European Mathematical Society (EMS), Zürich, 2008.

[2] Richard E. Borcherds. Coxeter groups, Lorentzian lattices, and K3 surfaces. Int. Math. Res. Not. IMRN, (19):1011-1031, 1998.

[3] Armand Borel and Jacques Tits. Groupes réductifs. Inst. Hautes Études Sci. Publ. Math. 27(1):55-150, 1965.

[4] Michel Brion. Personal communication. 2013.

[5] Winfried Bruns and Joseph Gubeladze. Polytopes, rings, and K-theory. Springer Monographs in Mathematics. Springer, Dordrecht, 2009.

[6] William A. Casselman. Geometric rationality of Satake compactifications. In Algebraic groups and Lie groups, volume 9 of Austral. Math. Soc. Lect. Ser., pages 81-103. Cambridge Univ. Press, Cambridge, 1997.

[7] Paola Cellini and Mario Marietti. Root polytopes and Borel subalgebras. Int. Math. Res. Not. IMRN, (12):4392-4420, 2015.

[8] Gurbir Dhillon and Apoorva Khare. Characters of highest weight modules and integrability. Preprint, arXiv:1606.09640, 2016.

[9] Xin Fang. Non-symmetrizable quantum groups: defining ideals and specialization. Publ. Res. Inst. Math. Sci., 50(4):663-694, 2014. 
GURBIR DHILLON AND APOORVA KHARE

[10] Branko Grünbaum. Convex polytopes, volume 221 of Graduate Texts in Mathematics. SpringerVerlag, New York, second edition, 2003. Prepared and with a preface by Volker Kaibel, Victor Klee and Günter M. Ziegler.

[11] Jin Hong and Seok-Jin Kang. Introduction to quantum groups and crystal bases, volume 42 of Graduate Studies in Mathematics. American Mathematical Society, Providence, RI, 2002.

[12] Jens C. Jantzen. Lectures on quantum groups, volume 6 of Graduate Studies in Mathematics. American Mathematical Society, Providence, RI, 1996.

[13] Victor G. Kac. Infinite-dimensional Lie algebras. Cambridge University Press, Cambridge, third edition, 1990.

[14] Joel Kamnitzer. Mirković-Vilonen cycles and polytopes. Ann. of Math. (2), 171(1):245-294, 2010.

[15] Masaki Kashiwara. On crystal bases. In Representations of groups (Banff, AB, 1994), volume 16 of CMS Conf. Proc., pages 155-197. Amer. Math. Soc., Providence, RI, 1995.

[16] David A. Kazhdan, Michael Larsen, and Yakov Varshavsky. The Tannakian formalism and the Langlands conjectures. Algebra Number Theory 8(1):243-256, 2014.

[17] Apoorva Khare. Faces and maximizer subsets of highest weight modules. J. Algebra, 455:32-76, 2016.

[18] Apoorva Khare. Standard parabolic subsets of highest weight modules. Trans. Amer. Math. Soc., 369(4):2363-2394, 2017.

[19] Shrawan Kumar. Kac-Moody groups, their flag varieties and representation theory, volume 204 of Progress in Mathematics. Birkhäuser Boston, Inc., Boston, MA, 2002.

[20] James Lepowsky. A generalization of the Bernstein-Gelfand-Gelfand resolution. J. Algebra, 49(2):496-511, 1977.

[21] Zhou Li, You'an Cao, and Zhenheng Li. Cross-section lattices of $\mathcal{J}$-irreducible monoids, and orbit structures of weight polytopes. Preprint, arXiv:1411.6140, 2014.

[22] Eduard Looijenga. Invariant theory for generalized root systems. Invent. Math., 61(1):1-32, 1980.

[23] Eduard Looijenga. Discrete automorphism groups of convex cones of finite type. Compos. Math., 150(11):1939-1962, 2014.

[24] George Lusztig. Quantum deformations of certain simple modules over enveloping algebras. Adv. Math. 70(2):237-249, 1988.

[25] Ichirô Satake. On representations and compactifications of symmetric Riemannian spaces. Ann. of Math. (2), 71:77-110, 1960.

[26] Ernest B. Vinberg. Some commutative subalgebras of a universal enveloping algebra. Izv. Akad. Nauk SSSR Ser. Mat., 54(1):3-25, 221, 1990.

(G. Dhillon) Department of Mathematics, Stanford University, Stanford, CA 94305, USA

E-mail address: gsd@stanford.edu

(A. Khare) Department of Mathematics, Indian Institute of Science, Bangalore 560012, INDIA

E-mail address: khare@math.iisc.ernet.in 